\title{
New Super-Selection Sectors ("Soliton-States") in Two Dimensional Bose Quantum Field Models
}

\author{
Jürg Fröhlich* \\ Department of Mathematics, Princeton University, Princeton, N. J. 08540, USA
}

\begin{abstract}
A rigorous construction of new super-selection sectors - so-called "soliton-sectors" - for the quantum "sine-Gordon" equation and the $(\phi \cdot \phi)^{2}$ quantum field models with explicitly broken isospin symmetry in two spacetime dimensions is presented. These sectors are eigenspaces of the charge $Q \equiv \int d x(\operatorname{grad} \phi)(x)$ with non-zero eigenvalue. The scattering theory for quantum solitons is briefly discussed and shown to have consequences for the physics in the vacuum sector. A general theory is developed which explains why soliton-sectors may exist for theories in two but not in four space-time dimensions except possibly for non-abelian Yang-Mills theories.
\end{abstract}

In quantum field theory a great deal of attention has recently been paid to the construction and analysis of new super-selection sectors orthogonal to the vacuum sector. Most authors - and this is not an accident (see Section 6) - have studied Bose quantum field models in two space-time dimensions such as the quantum "sine-Gordon" equation $[3,8,14,15]$ and the $\phi^{4}$-model $[4,8,21,35]^{1}$ which are known to exist and to define relativistic quantum field theories $[12,36,15,17]$. A deep axiomatic analysis of super-selection sectors in the framework of algebras of local observables has earlier been presented in [9]. (Some of the results of [9], e.g. the analysis of the statistics of a super-selection sector, do however not apply to two space-time dimensions.)

For the two dimensional models these new sectors are expected to contain states describing somewhat unusual collective phenomena which may be related to the "soliton"-solutions of the $c$-number, non-linear partial differential field equations, the "sine-Gordon" equation $[8,14]$, or the equation

$$
\left(\square+m^{2}\right) \phi(x, t)=-\lambda \phi(x, t)^{3}, m^{2}<0 ;
$$

\footnotetext{
* Supported in part by the National Science Foundation under Grant NSF-GP-39048 and by the ETH, Zürich.

1 See also Remark 7, Section 6.
} 
see $[21,8,35]^{1}$. See also $[31,14,35]$ and refs. given there for interesting results about these field equations. For such models this paper attempts to clarify on a mathematically rigorous basis the existence question of new sectors and of states with the property that the expectation value of the field operator in such states is similar to the classical soliton-solution (for this reason these states are called "soliton-states" in this paper).

Technically a soliton-sector is defined by the following two properties:

- It is space-time translation covariant.

- It is an eigenspace of the charge $\boldsymbol{Q} \equiv \int d x(\operatorname{grad} \phi)(x)$ with non-zero eigenvalue (here $\phi$ is an $n$-component, canonical scalar Bose field).

Our analysis intends to explain the general mechanism which is responsible for the occurrence of new sectors in two dimensional models: It is related to the spontaneous breaking of an internal symmetry of the dynamics (of determinant +1 ). We prove that, within the framework of the models considered in this paper, soliton-sectors are connected with the vacuum sector by certain Bogoiubov transformations. Some connections with the analysis of [9] and with results of [42] are explained.

Sections 1-5 are devoted to the analysis of specific models and their superselection sectors; Section 6 contains abstract conclusions derived from Sections 1-5: A general theory of "soliton-sectors".

In this paper we explain the main results and present or at least outline their proofs. Some of the technical details appear in forthcoming papers: $[15,20]$. Whereas we have written this paper following the natural order of thoughts we recommend that (for an overall view) the reader first read Sections 1, 2, 1', 2', and 6 and only then proceed to the more technical Sections 3-5.

We will mainly be concerned with the discussion of two specific models which we now define in terms of their formal Lagrangians.

\section{$\S 1$ The $\cos \varepsilon \phi_{2}$-Theory}

This model describes a neutral, scalar, relativistic quantum field $\phi$ in two spacetime dimensions. The total Lagrangian density is

$$
\mathscr{L}(x)=\mathscr{L}_{0}(x)-\lambda: \cos (\varepsilon \phi(x, 0)+\theta):
$$

where $\mathscr{L}_{0}(x)$ is the free Lagrangian density with bare mass $m=0$, the colons denote Wick ordering with respect to bare mass $1[3,15]$; the coupling constant $\lambda$ is an arbitrary, real number, and $\theta \in[0,2 \pi)$ an arbitrary angle.

This model has only been shown to exist for $\varepsilon^{2} \leqq 4 \pi$, $[15,16,17]$. If $\varepsilon^{2}=4 \pi$ it is easily seen to be equivalent to the one describing a free, massive Dirac field $[3,15]$. For $\varepsilon^{2}<4 \pi$ it has been shown to be isomorphic to the massive Thirring model [3]. For $\varepsilon^{2}>4 \pi$ it has non-trivial, non-superrenormalizable ultraviolet divergencies which are not well understood, yet, [15].

The $\cos \varepsilon \phi_{2}$-theory has been extensively discussed in [8] and from a mathematical point of view in $[15,17]$. The reason why we briefly discuss it in this paper again is that it is the simplest (and in some sense the only) model which has infinitely many (rather than only finitely many) super-selection sectors labeled by an integer charge. This is connected to the fact that $\mathscr{L}$ is invariant under shifts 
of the field $\phi$ by $2 m \pi \varepsilon^{-1}, m \in \mathbb{Z}$. The vacuum sector contains states of pairs of oppositely charged particles but no charged one particle states. Needless to say that the model is of some interest to physics, e.g. in non-linear optics [31]. Other (closely related) models of this category are discussed in [15].

\section{$\S$ 2. The $(\phi \cdot \phi)_{2}^{2}$-Theory}

Let $\phi=\left(\phi_{1}, \ldots, \phi_{n}\right)$ be an $n$-tuple of neutral, scalar fields and $\mathscr{L}_{0}$ the free Lagrangian density for $\phi$ with bare masses $m_{1}=\ldots=m_{n}=1$. The total Lagrangian density is

$$
\mathscr{L}(x)=\mathscr{L}_{0}(x)-\lambda:(\phi(x, 0) \cdot \phi(x, 0))^{2}:+\sum_{i=1}^{n} \sigma_{i}: \phi_{i}(x, 0)^{2}: ;
$$

$\mathscr{L}$ is invariant under the $n$ substitutions $\phi_{i} \mapsto-\phi_{i}, i=1, \ldots, n$. The mass terms $\sigma_{i}: \phi_{i}(x, 0)^{2}$ : break the $O(n)$-invariance, unless $\sigma_{1}=\ldots=\sigma_{n}$. As we will see this theory has only non-trivial super-selection rules if some sub-symmetry group of the symmetry group $O(n)$ is spontaneously broken. If $\sigma_{1}=\ldots=\sigma_{n}$ the theory is $O(n)$-invariant and, as a consequence of the Goldstone theorem [13], no such sub-symmetry group can be broken in this theory. For, it is obvious that the spontaneous breaking of a discrete sub-symmetry group (which is possible in two dimensions) would imply the breaking of $O(n)$ : some polynomial in $\phi$ not invariant under $O(n)$ would necessarily develop a non-vanishing vacuum expectation value which proves our assertion. This however is impossible in two space-time dimensions [13]. Hence, in order for the $(\boldsymbol{\phi} \cdot \boldsymbol{\phi})_{2}^{2}$-theory to have non-trivial superselection rules, at least one of the coefficients of the mass terms must be different from the others. If $\sigma_{1} \neq \sigma_{i}, i=2, \ldots, n$, the $\phi_{1} \mapsto-\phi_{1}$ symmetry may be broken spontaneously, and this is actually to be expected if $\sigma_{1} \gg \sigma_{i}, i=2, \ldots, n$, on the basis of the classical Goldstone picture or an approximate calculation of the effective potential. A rigorous proof follows from recent results of Glimm, Jaffe, and Spencer [28] and will be presented elsewhere. If the $\phi_{1} \mapsto-\phi_{1}$ symmetry is spontaneously broken we are able to construct (at least) two non-trivial superselection sectors consisting of soliton states; see Theorems 3 and 4 . The existence of soliton states is related to the invariance of $\mathscr{L}$ under the following substitutions of determinant +1 :

$$
\left(\phi_{1}, \ldots, \phi_{n}\right) \mapsto\left(-\phi_{1}, \ldots, e_{n} \phi_{n}\right), \text { where } e_{j}=-1,
$$

for some $j$, and $e_{i}=1$, for $i \neq 1, j$. Soliton-states occur if one of these symmetries is spontaneously broken.

If the classical Goldstone potential $(\boldsymbol{x} \cdot \boldsymbol{x})^{2}-\sum_{i=1}^{n} \sigma_{i} x_{i}^{2}$ has only finitely many absolute minima, it has at most two. We therefore expect that the $\phi_{i} \mapsto-\phi_{i^{-}}$ symmetry is broken for at most one $i$ and hence that there exist no more than two non-trivial super-selection sectors. Without loss of generality we may therefore set $n=2, \sigma_{1}=\sigma, \sigma_{2}=0$ throughout most of the rest of this paper. A more general situation is met if one considers e.g. a $(\boldsymbol{\phi} \cdot \boldsymbol{\phi})_{2}^{m}$-theory or the coupling of several independent systems with broken symmetries.

In a $P(\phi)_{2}$-model with one single, neutral, scalar field $\phi$ the substitution $\phi \mapsto-\phi$ has determinant -1 . We show in Section 5 that this forces us to consider 
a tensor product theory of two identical copies of the usual $P(\phi)_{2}$-theory which has an internal symmetry of determinant +1 . Starting from the (artificial) tensor product theory we are able to construct soliton sectors for the ordinary $P(\phi)_{2}$ theory. Our construction is quite different from recent more heuristic proposals.

The $(\phi \cdot \phi)_{2}^{2}$-theory is a model for a one-dimensional anharmonic, anisotropic dielectric chain: The field $\phi$ (with two or three components) is then interpreted as the polarization field which is bound by anharmonic, anisotropic $\left(\sigma_{1} \gg \sigma_{2}=0\right.$ !) forces. In order to understand what "soliton-states" are we must consider the effect of a spatially extended twist (torsion) of the dipole chain by a total angle $\pi$. Our techniques extend to the case where $\phi$ is coupled to some modes of the electric field. The expectation value of the electric field in a soliton-state has the shape of a pulse. Such a model may be relevant in quantum optics.

We now discuss the existence of a vacuum sector for the $\cos \varepsilon \phi_{2}$-theory with $\varepsilon^{2}<16 / \pi$ and the $(\phi \cdot \phi)_{2}^{2}$-theory.

Theorem 1. (a) For the $\cos \varepsilon \phi_{2}$-theory with $\varepsilon^{2}<16 / \pi$ there exists a vacuum sector $\mathscr{H}$ ( $\mathscr{H}$ is a separable Hilbert space) such that the theory on the vacuum sector satisfies all Wightman axioms [30] with the possible exception of uniqueness of the vacuum.

(b) For $\phi=\left(\phi_{1}, \ldots, \phi_{n}\right), n=1,2$ or $3, \sigma_{1} \equiv \sigma \geqq 0, \sigma_{2}=\sigma_{3}=0$ there exists a vacuum sector $\mathscr{H}$ for the $(\phi \cdot \phi)_{2}^{2}$-theory such that on $\mathscr{H}$ this theory satisfies all Wightman axioms with the possible exception of uniqueness of the vacuum. (For $0 \leqq \lambda<\lambda_{0}$, $\sigma<\sigma_{0}$, where $\lambda_{0}$ and $\sigma_{0}$ are small positive numbers, the vacuum is unique and there exists a mass gap and one particle states.)

(c) Under the assumptions of part (b) the $(\phi \cdot \phi)_{2}^{2}$-Wightman theory on the vacuum sector $\mathscr{H}$ of part (b) coincides with the theory obtained from the $C^{*}$-algebra construction of Glimm and Jaffe [22, 24]; see also Section 3.

Proof. (a) is proved in detail in $[15,16]$ (the restriction $\varepsilon^{2}<16 / \pi$ rather than $\varepsilon^{2}<4 \pi$ is presumably an artefact of our way of estimating).

For $n=1(\mathrm{~b})$ is well known. For a proof see [36] and refs. given there. For further results see $[37,20]$. For $n=2,3$ the proof of $(b)$ is due to the author [20]. It is based on Spencer's "large external field" expansion [38] which yields existence of the $\left[(\phi \cdot \phi)^{2}-\sigma \phi_{1}^{2}-\mu \phi_{1}\right]_{2}$-model for large $|\mu|$. The Lee-Yang theorems of [39] and [11] permit us to continue the Euclidean Green's functions of the model analytically in $\mu$ to arbitrary $\mu \neq 0$ and to construct limits as $\mu \searrow 0, \mu \rtimes 0$. The part of (b) within brackets (weak coupling) follows from the cluster expansion $[12,38]$. Part (c) follows from combining (b) with the $\phi$-bounds of $[20]-$ see also $[25,26]-$ which yield selfadjointness of the quantum fields on $\mathscr{H}$ and then applying a general result of [26]. Q.E.D.

Remarks. We would like to emphasize that the $C^{*}$-algebra construction of quantum fields in two space-time dimensions as developed in $[23,24]$ is particularly useful for the purposes of this paper: the analysis of superselection rules.

Theorem 1, (b) has various generalizations including simple proofs of existence and analyticity for lattice systems such as the Heisenberg model, [20].

Since the time when this manuscript was completed Glimm, Jaffe and Spencer [28] have established spontaneous $\phi \rightarrow-\phi$ symmetry breaking under the conditions of Theorem $1,(\mathrm{~b}), \sigma \gg 1, n=1$. Thanks to correlation inequalities proved in 
[11] and results of [20] the author could extend this result to the $(\phi \cdot \phi)_{2}^{2}$-theory with $\sigma \gg 1$ and $n=2$. This removes the last unproved assumption in Sections $2^{\prime}$ and 3 .

For the $\cos \varepsilon \phi_{2}$-theory some interesting, heuristic results on the mass spectrum are presented in $[8]$. In $[15,16]$ it is proved that the $\cos \varepsilon \phi_{2}$-theory is mathematically isomorphic to the theory of the classical, two-component, neutral Coulomb gas in two space dimensions. The well-known scaling properties of the Coulomb gas [33] are used in [16] to prove ${ }^{2}$ :

$$
\begin{aligned}
p(\alpha, \lambda) & =F(\alpha)|\lambda|^{2 /(2-\alpha)}, m_{*}(\alpha, \lambda)=G(\alpha)|\lambda|^{1 /(2-\alpha)}, \\
\vartheta^{2 \alpha} u_{\vartheta^{\alpha-2 \lambda}}^{(2)}(x, t) & =u_{\lambda}^{(2)}\left(\vartheta^{-1} x, \vartheta^{-1} t\right), \vartheta \in(0, \infty),
\end{aligned}
$$

where $p(\alpha, \lambda)$ is the vacuum energy density, $m_{*}(\alpha, \lambda)$ the physical mass and $u_{\lambda}^{(2)}(x, t)$ the truncated two point Euclidean Green's function of the field: $e^{i \varepsilon \phi}:(x, t)$ of the $\cos \varepsilon \phi_{2}$-theory; $\alpha=\varepsilon^{2} / 4 \pi$. Scaling equations similar to (5) hold for all the truncated $n$-point Euclidean Green's functions.

Notice that for $\alpha \neq 1 p, m_{*}$ and $u^{(2)}$ have a branch point singularity at $\lambda=0$. For $\alpha<1$ this singularity is caused by infrared divergencies (second order perturbation theory is infrared divergent). For $\alpha>1$ the singularity is caused by ultraviolet divergencies. Equations (4) imply that for $\alpha>1$ the $\cos \varepsilon \phi_{2}$-theory is not superrenormalizable, and for $\alpha \geqq 2$ it is presumably meaningless (see also [3]). By the equivalence of $\cos \varepsilon \phi_{2}$ with the massive Thirring model Eq. (5) proves that the short distance singularities of the massive Thirring model are identical to the ones of the massless Thirring model.

The isomorphism between $\cos \varepsilon \phi_{2}$ and the classical Coulomb gas suggests that the physical mass $m_{*}$ is positive. This is interpreted as Debye screening in the Coulomb gas. One knows that $G(\alpha=0)^{-1}=0$ and $G(\alpha=1)^{-1}=0$ or 2 -depending on the choice of the renormalization scheme - see [16]. This suggests that $G(\alpha)^{-1}$ is bounded for $\alpha$ sufficiently close to 0 or 1 and therefore $m_{*}(\alpha, \lambda)>0$ for all real $\lambda \neq 0$, i.e., the mass gap is positive, for $\lambda \neq 0$.

Next we describe our construction of new super-selection sectors for the theories described in Sections 1, 2, and Theorem 1.

\section{$\S 1^{\prime}$. The Sectors of the $\cos \varepsilon \phi_{2}$-Theory}

In the discussion of the $\cos \varepsilon \phi_{2}$-theory we choose as our basic observables the following selfadjoint fields [15]:

$$
: \cos [\varepsilon \phi(x, t)+\beta]:, \beta \in[0,2 \pi), \partial_{x} \phi(x, t), \pi(x, t),
$$

where $\partial_{x}$ denotes derivative in $x$ and $\pi$ is the momentum operator canonically conjugate to $\phi$. Note that $\partial_{x} \phi(x, t)$ is the density of a conserved charge

$$
Q=(\varepsilon / 2 \pi) \int d x \partial_{x} \phi(x, t) ; \quad \text { see }[3,15] \text {. }
$$

Let $\mathfrak{A}(\mathcal{O})$ denote the usual local von Neumann algebra $[9,22]$ generated by the bounded functions of the fields defined in (6) smeared with test functions that are

\footnotetext{
In order to prove the equation for $m_{*}$ and (5) we must assume that $m_{*}(\alpha, \lambda)>0$, for some $\lambda \neq 0$.
} 
supported in a bounded open region $\mathscr{O} \subset \mathbb{R}^{2}$ (e.g. a diamond). We let $\mathfrak{A}$ be the $C^{*}$-algebra of all local observables [2] obtained by taking the closure of $\bigcup_{\{\mathcal{O}\}} \mathfrak{H}(\mathcal{O})$ in the operator norm, where $\{\mathcal{O}\}$ is a covering of $\mathbb{R}^{2}$ by bounded, open diamonds.

We set $\phi(x) \equiv \phi(x, 0)$ and $\pi(x) \equiv \pi(x, 0)$. A local *automorphism $\varrho_{g}$ of $\mathfrak{U}$ is defined by

$$
\left.\begin{array}{rl}
\varrho_{g}(: \cos [\varepsilon \phi(x)+\beta]:) & =: \cos [\varepsilon(\phi(x)+g(x))+\beta]: \\
\left.\varrho_{g}\left(\partial_{x} \phi(x)\right)\right) & =\partial_{x}(\phi(x)+g(x)) ; \varrho_{g}(\pi(x))=\pi(x) .
\end{array}\right\}
$$

It is known, though not quite trivial to prove that the automorphism $\varrho_{g}$ is determined on all of $\mathfrak{A}$ if we know how it acts on the time 0-fields, i.e. by (8); see [15]. Here we let $g(x)$ be a continuously differentiable function on the real line with

$$
\lim _{x \rightarrow-\infty} g(x)=0, \lim _{x \rightarrow+\infty} g(x)=g_{\infty}, \operatorname{supp}\left(\partial_{x} g\right) \quad \text { compact . }
$$

Such a function is called a kink function. When restricted to $\mathfrak{Q}(\mathcal{O}), \mathcal{O} \in\{\mathcal{O}\}, \varrho_{g}$ is unitarily implemented by $e^{i \pi\left(g_{\theta}\right)}$, where $g_{\mathcal{O}}$ is a continuously differentiable function with $g_{\mathcal{O}}(x)=g(x)$ on $\mathcal{O}, \operatorname{supp} g_{\mathcal{O}}$ compact. The operator intertwining the representations of $\mathfrak{A}$ and of $\varrho_{g}(\mathfrak{R})$ on $\mathscr{H}$ [i.e. the representations of $\mathfrak{A}$ on $\mathscr{H}$ and on the Hilbert space obtained from $\left(\mathfrak{Q},\left\langle\Omega, \varrho_{g}(\cdot) \Omega\right\rangle\right)$, where $\Omega$ is the vacuum in $\mathscr{H}$, by the Gelfand-Naimark-Segal construction] is denoted by $T_{g}$. Formally $T_{g}=e^{i \pi(g)}$. Under the automorphism $\varrho_{g}$ the dynamics of the $\cos \varepsilon \phi_{2}$-theory transforms as follows:

$$
\varrho_{g}\left(e^{i t H}\right)=e^{i t(H+\delta H(g))},
$$

where

$$
\begin{aligned}
\delta H(g)= & \partial_{x} \phi\left(\partial_{x} g\right)+\frac{1}{2}\left\|\partial_{x} g\right\|_{2}^{2} \\
& +\lambda \int d x[: \cos (\varepsilon \phi(x)+\theta):(\cos \varepsilon g(x)-1) \\
& +: \cos (\varepsilon \phi(x)+\theta-\pi / 2): \sin \varepsilon g(x)] .
\end{aligned}
$$

Inspection of the r.h.s. of (11) shows that $\delta H(g)$ is a tiny form perturbation of the Hamiltonian $H$ if and only if $(\cos \varepsilon g(x)-1)$ and $\sin \varepsilon g(x)$ have compact support, i.e.

$$
\varepsilon g_{\infty}=2 m \pi, m \in \mathbb{Z}, \quad \text { ("soliton-condition"). }
$$

The proof of this assertion is based on a straightforward generalization of the Glimm-Jaffe $\phi$-, : $\phi^{j}:-$, and $\partial_{x} \phi$-bounds $[24,25,26]$ for the $\left(\cos \varepsilon \phi+m^{2} \phi^{2}\right)_{2}$ theories, for all $m \geqq 0$, and is given in [15]. Clearly the : $\phi^{j}$ :-bounds of the $P(\phi)_{2^{-}}$ models are replaced in the $\cos \varepsilon \phi_{2}$-theory by $: \cos (\varepsilon \phi+\beta)$ :-bounds.

On a formel level (11) is an immediate consequence of (1), (8), and (10). The rigorous proof of (11) involves first studying the $\left(\cos \varepsilon \phi+m^{2} \phi^{2}\right)_{2}$-theory $[15,17]$ and the automorphism $\varrho_{g-g_{\xi}}$, where $g$ satisfies (12) and $g_{\xi} \equiv g(x-\xi)$. Then $g-g_{\xi}$ has compact support, and $\varrho_{g-g_{\xi}}$ is unitarily implemented on $\mathscr{H}$ by $e^{i \pi\left(g-g_{\xi}\right)}$. The proof of (11) with $g$ replaced by $g-g_{\xi}$ is then quite easy. One then lets $m$ tend to 0 , decomposes the limiting theory into its pure phases - using a theorem of [19] and then lets $\xi$ tend to $\infty$. This limit exists because of the cluster properties of a 
pure phase theory (it is interpreted as "sending the anti-soliton to behind the moon"). For details see [15]. Using the quadratic form estimates described above and path space techniques one can show that

$$
e^{i t(H+\delta H(g))} \varrho_{g}(A) e^{i t(H+\delta H(g))}=\varrho_{g}\left(e^{i t H} A e^{i t H}\right), \quad \text { all } \quad A \in \mathfrak{A},
$$

where $H+\delta H(g)$ is the Friedrichs extension of the corresponding quadratic form. We have now sketched the proof of

Theorem 2. All vectors of the form $\left\{T_{g} \Psi \mid \Psi \in \mathscr{H}, \varepsilon g_{\infty}=2 m \pi\right\}$ span a new Hilbert space $\mathscr{H}_{m}$. The time-translation automorphisms of $\mathfrak{A}$ are implemented on $\mathscr{H}_{m}$ by a continuous unitary group $\left\{e^{i t H} / t \in \mathbb{R}\right\}$ :

$$
e^{i t H} T_{g} \Psi=T_{g} e^{i t(H+\delta H(g))} \Psi, \text { all } \Psi \in \mathscr{H} ;
$$

and

$$
Q T_{g} \Psi=m T_{g} \Psi
$$

For all vectors $\Psi$ in a dense subspace of $\mathscr{H}$

$$
\lim _{x \rightarrow+\infty}\left\langle T_{g} \Psi, \phi(x, t) T_{g} \Psi\right\rangle=\lim _{x \rightarrow-\infty}\left\langle T_{g} \Psi, \phi(x, t) T_{g} \Psi\right\rangle+2 m \pi
$$

(the limits on both sides of (15) exist; $\|\Psi\|=1$ ). For $m \neq n$ the representations of $\mathfrak{A}$ on $\mathscr{H}_{m}$ and $\mathscr{H}_{n}$ are unitarily inequivalent, i.e. $\mathscr{H}_{m}$ and $\mathscr{H}_{n}$ are orthogonal sectors.

Remarks. (14) is an immediate consequence of (7) and (8). Concerning (15) notice that

and

$$
\begin{aligned}
\lim _{x \rightarrow+\infty}\left\langle T_{g} \Psi, \phi(x, t) T_{g} \Psi\right\rangle & =\lim _{x \rightarrow+\infty}\left\langle T_{g} \Psi, \phi(x) T_{g} \Psi\right\rangle, \text { by (13), } \\
& =\lim _{x \rightarrow+\infty}\left\langle\Psi, \varrho_{g}(\phi(x)) \Psi\right\rangle=\lim _{x \rightarrow+\infty}\langle\Psi, \phi(x) \Psi\rangle+2 m \pi,
\end{aligned}
$$

$$
\begin{aligned}
\lim _{x \rightarrow-\infty}\left\langle T_{g} \Psi, \phi(x, t) T_{g} \Psi\right\rangle=\ldots & =\lim _{x \rightarrow-\infty}\left\langle\Psi, \varrho_{g}(\phi(x)) \Psi\right\rangle \\
& =\lim _{x \rightarrow-\infty}\langle\Psi, \phi(x) \Psi\rangle=\lim _{x \rightarrow+\infty}\langle\Psi, \phi(x) \Psi\rangle .
\end{aligned}
$$

The orthogonality of $\mathscr{H}_{m}$ and $\mathscr{H}_{n}$ for $m \neq n$ is a direct consequence of (15). (Heuristically it also follows from the selfadjointness of $Q$ on the total Hilbert space $\bigoplus_{m \in \mathbb{Z}} \mathscr{H}_{m}$, the spectral theorem and (14).) The rigorous proof follows slightly different lines; see [15]. It is easy to show that for all $m$ the space-translations of $\mathfrak{A}$ are unitarily implemented on $\mathscr{H}_{m}$.

States in $\mathscr{H}_{m}$, for $m \neq 0$, are interpreted as "soliton"- $(m>0)$ and "anti-soliton"$(m<0)$ states; $\left\langle T_{g} \Psi, \phi(x, t) T_{g} \Psi\right\rangle$ seems to be related to the soliton-solutions $[14,8]$ of the classical "sine-Gordon" equation.

The super-selection sectors $\mathscr{H}_{m}$ must be identical with the charged sectors of the massive Thirring model [3]. It is an interesting open problem to construct local fields with non-vanishing matrix elements between $\mathscr{H}_{m}$ and $\mathscr{H}_{m+1}$ : the Fermion fields of the massive Thirring model.

In the massless Thirring model there are uncountably many super-selection sectors labeled by a pair $\left(g_{\infty}, h_{\infty}\right)$, where $h_{\infty}$ is a real number [42]. There is no restriction on the value of $g_{\infty}$. The "soliton-condition" (12) can thus be interpreted as a dynamical charge quantization (which is enforced by the mass of the Fermions). 


\section{$\S 2^{\prime}$. The Sectors of the $(\phi \cdot \phi)_{2}^{2}$-Theory}

Since we have broken $O(2)$-invariance in this theory explicitly by a mass term, we have no reason to restrict the algebra of local observables to $O(2)$-invariant (bounded) operators. We may therefore choose as our basic observable fields the selfadjoint time 0 -fields

$$
\phi_{1}(x), \pi_{1}(x), \phi_{2}(x) \text { and } \pi_{2}(x),
$$

where $\pi_{j}$ is the momentum canonically conjugate to $\phi_{j}, j=1,2$.

Let $\mathfrak{A}(\Lambda)$ be the usual local von Neumann algebra generated by all bounded functions of $\phi_{1}, \pi_{1}, \phi_{2}$, and $\pi_{2}$ smeared with test functions supported on a compact interval $\Lambda$ of the real line $(\mathfrak{U}(\Lambda)$ is identical with the local von Neumann algebra $\mathfrak{H}(\mathcal{O})$, where $\mathcal{O}$ is the diamond with base $\Lambda$, and $\mathfrak{H}(\mathcal{O})$ is defined in the usual manner, [22]).

We let $\mathfrak{A}$ be the $C^{*}$-algebra obtained by taking the closure of $\bigcup_{\{\Lambda\}} \mathfrak{A}(\Lambda)$ in the operator norm ( $\{\Lambda\}$ is a covering of the real line by compact intervals). The construction of the algebra $\mathfrak{A}(\Lambda), \Lambda \in\{\Lambda\}$, can be done on the Fock space of the free, charged scalar field $\phi$ because of the "locally-Fock" property - proved in [23] for the $\phi_{2}^{4}$-model - which holds for the $(\phi \cdot \phi)_{2}^{2}$-theory, as well.

Let $\alpha$ be a continuously differentiable, bounded function on the real line.

The following equations define a ${ }^{*}$-automorphism $\varrho_{\alpha}$ of the algebra $\mathfrak{Q}$ :

$$
\left.\begin{array}{l}
\varrho_{\alpha}\left(\phi_{1}(x)\right)=\cos \alpha(x) \cdot \phi_{1}(x)+\sin \alpha(x) \cdot \phi_{2}(x) \\
\varrho_{\alpha}\left(\phi_{2}(x)\right)=-\sin \alpha(x) \cdot \phi_{1}(x)+\cos \alpha(x) \cdot \phi_{2}(x), \\
\text { plus identical equations for } \pi_{1}(x) \text { and } \pi_{2}(x) .
\end{array}\right\}
$$

For $g$ a continuously differentiable, integrable function on the real line we define

$$
L(g) \equiv \int d x\left[\phi_{1}(x) \pi_{2}(x)-\pi_{1}(x) \phi_{2}(x)\right] g(x) .
$$

Obviously $L\left(\delta_{x}\right)$ is the charge density. On the Fock space of the free, charged scalar field $L(g)$ is known to define a selfadjoint operator (also denoted by $L(g)$ ), provided $g$ is real; then $e^{i L(g)} \in \mathfrak{A}(\operatorname{supp} g)$. This is shown in Lemma 1 , below.

It then follows from the "locally-Fock" property that $e^{i L(g)}$ is unitary on the vacuum sector $\mathscr{H}$, for $g$ real and of compact support.

From the theory of quantum mechanical angular momentum or of the free, charged scalar field we know that, formally, $\varrho_{\alpha}$ is implemented by $e^{i L(\alpha)}$. Given $\Lambda \in\{\Lambda\}$, we can choose a function $\alpha_{\Lambda}$ with

$$
\operatorname{supp} \alpha_{A} \text { compact and } \alpha_{A} \equiv \alpha \text { on } \Lambda \text {. }
$$

The local action of $\varrho_{\alpha}$ and $e^{i L\left(\alpha_{A}\right)}-$ see Lemma 1, below - and the "locally-Fock" property then imply that for all $x \in \Lambda$

$$
\begin{aligned}
& \varrho_{\alpha}\left(\phi_{j}(x)\right)=e^{i L\left(\alpha_{\Lambda}\right)} \phi_{j}(x) e^{-i L\left(\alpha_{\Lambda}\right)} \\
& \varrho_{\alpha}\left(\pi_{j}(x)\right)=e^{i L\left(\alpha_{\Lambda}\right)} \pi_{j}(x) e^{-i L\left(\alpha_{\Lambda}\right)},
\end{aligned}
$$


$j=1,2$, in the sense of operator-valued distributions on $\mathscr{H}$. Therefore $\varrho_{\alpha}$ has a unique extension to the von Neumann algebras $\mathfrak{A}(\Lambda), \Lambda \in\{\Lambda\}$ :

$$
\varrho_{\alpha}(A)=e^{i L\left(\alpha_{\Lambda}\right)} A e^{-i L\left(\alpha_{\Lambda}\right)}, \text { for all } A \in \mathfrak{U}(\Lambda),
$$

[i.e. $\varrho_{\alpha} \uparrow \mathfrak{A}(\Lambda)$ is unitarily implemented by $e^{i L\left(\alpha_{\Lambda}\right)}$ ].

We conclude that $\varrho$ is a well-defined *-automorphism of $\bigcup_{\{\Lambda\}} \mathfrak{A}(\Lambda)$ and hence can be extended to a *automorphism of $\mathfrak{A}$ by continuity. Let $\Omega$ be the vacuum in $\mathscr{H}$ and set

$$
\omega(A)=\langle\Omega, A \Omega\rangle,
$$

and

$\omega^{\circ} \varrho_{\alpha}(A)=\omega\left(\varrho_{\alpha}(A)\right)$, for all $A \in \mathfrak{U}$.

The vacuum sector $\mathscr{H}$ of the $(\phi \cdot \phi)_{2}^{2}$-theory is the Hilbert space obtained from $(\mathfrak{I}, \omega)$ by the Gelfand-Naimark-Segal (G.N.S.) construction $[23,32]$. We denote the Hilbert space obtained from $\left(\mathfrak{A}, \omega^{\circ} \varrho_{\alpha}\right)$ by G.N.S. construction by $\mathscr{H}_{\alpha}$. The representations of $\mathfrak{U}$ on $\mathscr{H}$ and $\mathscr{H}_{\alpha}$ are intertwined by an isometric mapping

$$
T_{\alpha}: \mathscr{H} \rightarrow \mathscr{H}_{\alpha} .
$$

We may therefore write $\left\langle T_{\alpha} \Omega, \cdot T_{\alpha} \Omega\right\rangle$ for $\omega^{\circ} \varrho_{\alpha}(\cdot)$, etc.

Space-Time Translations. It is an important result due to Glimm and Jaffe [22] - they consider the $\phi_{2}^{4}$-model, but their results extend to $(\phi \cdot \phi)_{2}^{2}-$ that for arbitrary $A \in \mathfrak{U}$

$$
e^{i(t H-x P)} A e^{-i(t H-x P)} \text { is in } \mathfrak{U},
$$

so that

$$
\tau_{t, x}(A) \equiv e^{i(t H-x P)} A e^{-i(t H-x P)}
$$

defines a *-automorphism group (the space-time translations) on $\mathfrak{A}$. In the following a detailed analysis of the space-translations $\tau_{0, x}(x \in \mathbb{R})$ may mostly be omitted, since it is trivial. We may then write $\tau_{t}$ for $\tau_{t, 0}$.

Definition 0 . Let $\alpha^{+}$be a continuously differentiable, bounded function on the real line with

$$
\left.\begin{array}{l}
\lim _{x \rightarrow-\infty} \alpha^{+}(x)=0, \lim _{x \rightarrow+\infty} \alpha^{+}(x) \equiv \alpha_{\infty}=\pi \\
\operatorname{supp}\left(\partial_{x} \alpha^{+}\right) \text {compact } .
\end{array}\right\}
$$

We set $\alpha^{-}(x) \equiv \alpha^{+}(-x) ; \alpha_{x}^{ \pm}(y) \equiv \alpha^{ \pm}(y-x)$. For reasons that will become clear shortly we call (21) the "soliton-condition"

We now state our main result:

Theorem 3. All vectors of the form $\left\{T_{\alpha^{ \pm}} \Psi / \Psi \in \mathscr{H}, \alpha_{\infty}=\pi\right\}$ span a Hilbert space $\mathscr{H}_{\pi}^{ \pm}$. 
The space-time translation automorphisms of the algebra $\mathfrak{A}$ of all local observables are implemented on $\mathscr{H}_{\pi}^{ \pm}$by a strongly continuous, unitary group

$$
\left\{e^{i\left(t H_{\pi}^{ \pm}-x P_{\pi}^{ \pm}\right)} /(t, x) \in \mathbb{R}^{2}\right\}
$$

with

$$
e^{i t H_{\pi}^{ \pm}} T_{\alpha^{ \pm}} \Psi=T_{\alpha^{ \pm}} e^{i t H\left(\alpha^{ \pm}\right)} \Psi,
$$

where $H\left(\alpha^{ \pm}\right)$is a positive, selfadjoint operator on $\mathscr{H}$, and

$$
e^{i x P_{\pi}^{ \pm}} T_{\alpha^{ \pm}} \Psi=T_{\alpha^{ \pm}} e^{i L\left(\alpha_{x}-\alpha\right)} e^{i x P} \Psi ;
$$

moreover

$$
e^{i x P_{\pi}^{ \pm}} e^{i t H_{\pi}^{ \pm}}=e^{i t H_{\pi}^{ \pm}} e^{i x P_{\pi}^{ \pm}}
$$

Before we turn to the proof of Theorem 3 we want to make some remarks and indicate its somewhat surprising consequences.

Remarks. (1) The first part (G.N.S. construction of $\left.\mathscr{H}_{\pi}^{ \pm}\right),(23)$ and (24) are the easy portions of Theorem 3. The hard part is (22).

(2) Theorem 3 also applies to the Hilbert spaces spanned by $\left\{T_{\alpha^{ \pm}} \Psi / \Psi \in \mathscr{H}\right.$, $\left.\alpha_{\infty}=2 m \pi\right\}$ and $\left\{T_{\alpha^{ \pm}} \Psi / \Psi \in \mathscr{H}, \alpha_{\infty}=(2 m+1) \pi\right\}, m \in \mathbb{Z}$. But these are of course identical to $\mathscr{H}, \mathscr{H}_{\pi}^{ \pm}$, respectively. We come back to this point below.

(3) As one might guess now we expect that the Hilbert spaces $\mathscr{H}_{\pi}^{ \pm}$represent new super-selection sectors consisting of "soliton states". It turns out that in order to decide whether $\mathscr{H}_{\pi}^{ \pm}$is orthogonal to the vacuum sector $\mathscr{H}$ or not (i.e. whether the representations of $\mathfrak{U}$ on $\mathscr{H}_{\pi}^{ \pm}$and $\mathscr{H}$ are inequivalent - more precisely disjoint or not) we must know whether or not the $\phi_{1} \mapsto-\phi_{1}$ symmetry of the $(\phi \cdot \phi)_{2}^{2}$ Lagrangian is spontaneously broken on $\mathscr{H}$.

$\phi_{1} \mapsto-\phi_{1}$ symmetry breaking is predicted for sufficiently large $\sigma$ on the basis of the classical Goldstone picture. A proof follows from recent results of [28].

Let $\mathscr{H}_{p}$ be the vacuum sector of a pure phase $(\phi \cdot \phi)_{2}^{2}$-theory (i.e. the vacuum $\Omega_{p}$ in $\mathscr{H}_{p}$ is unique) with the $\phi_{1} \mapsto-\phi_{1}$ symmetry spontaneously broken. If this symmetry is broken there exist at least (and presumably only) two such sectors.

We may assume at this point - see however Theorem 4 - that

$$
\left\langle\Omega_{p}, \phi_{1}(x, t) \Omega_{p}\right\rangle \equiv \phi_{c}>0 .
$$

Then

$$
\begin{aligned}
& \left\langle T_{\alpha^{ \pm}} \Omega_{p}, \phi_{1}(x, t) T_{\alpha^{ \pm}} \Omega_{p}\right\rangle=\left\langle\Omega_{p}, \varrho_{\alpha^{ \pm}}\left(\phi_{1}(x, t)\right) \Omega_{p}\right\rangle \\
& =\left\langle e^{-i H\left(\alpha^{ \pm}\right) t} \Omega_{p}, \varrho_{\alpha^{ \pm}}\left(\phi_{1}(x)\right) e^{-i H\left(\alpha^{ \pm}\right) t} \Omega_{p}\right\rangle \\
& \rightarrow\left\{\begin{array}{lll}
\mp \phi_{c}, & \text { as } & x \rightarrow+\infty, \\
\pm \phi_{c}, & \text { as } & x \rightarrow-\infty
\end{array}\right.
\end{aligned}
$$

From (25) one can deduce that $\mathscr{H}_{\pi}^{ \pm}$is orthogonal to $\mathscr{H}_{p}$ and that the spectrum of $P_{\pi}^{ \pm}$is purely continuous; see Theorem 4 .

It is rather striking how much the functions $\left\langle T_{\alpha^{ \pm}} \Omega_{p}, \phi_{1}(x, t) T_{\alpha^{ \pm}} \Omega_{p}\right\rangle$ resemble soliton-solutions of the classical field equations even though they are in general not strictly stationary in $t$. 


\section{§ 3. Proof of Theorem 3 and More about the Solitons in $(\phi \cdot \phi)_{2}^{2}$}

Proof of Theorem 3. The proof is given in seven (rather lengthy) steps (Lemmata $1-7)$.

A general comment on the strategy of our proof seems to be appropriate: The construction of the vacuum sector of the $(\phi \cdot \phi)_{2}^{2}$-theory in the infinite volume limit is based on Euclidean field theory methods and the Lee-Yang theorem $[39,11]$ which yield convergence and analyticity properties in bare parameters of the Euclidean Green's functions with periodic boundary conditions ("periodic box-cutoff'). This construction (see Theorem 1) is given in [20]. It is expected to automatically yield pure phase theories. (The main technical advantage of periodic boundary conditions is that they preserve space-translation invariance at each stage. See $[24,27]$ for an analysis of boundary conditions in the $P(\phi)_{2}$-quantum field models.)

Our construction of soliton sectors and the dynamics on these sectors is however based in an essential manner on $C^{*}$-algebra techniques developed earlier by Glimm and Jaffe [23, 24]. We have two reasons for doing so:

- Although the input we are using might not be very well known to this or the other reader the $C^{*}$-algebra techniques are very adequate to the problem we are studying and they yield elegant proofs.

- They permit us to use powerful techniques such as the "locally-Fock" property, finite propagation speed [22,23], duality (for the free field only) [34] which in a sense have still no equivalent Euclidean counterpart. It is however quite clear that our main results could be proved in a purely Euclidean setting - as it is the case in the $\cos \varepsilon \phi_{2}$-theory (Theorem 2) [15] - mainly based on path space techniques and the convergence of the lattice approximation [27]. This assertion can be checked easily for a $(\boldsymbol{\phi} \cdot \boldsymbol{\phi})_{2}^{2}$-theory on a lattice.

Definition 1. Let $\mathscr{F}$ denote the usual Fock space of the free, charged scalar field, and

$$
\mathscr{F}_{V} \equiv\left(\bigoplus_{m=0}^{\infty} L^{2}([-V / 2, V / 2], d x)^{\otimes_{s} m}\right)^{\otimes 2}
$$

the Fock space of the free, charged scalar field $\phi_{V}$ with periodic boundary conditions at $x= \pm V / 2$. The momentum operator canonically conjugate to $\phi_{V}$ is denoted by $\pi_{V}$. For $\Lambda$ a finite union of closed intervals (contained in $[-V / 2, V / 2]$ ) $\mathfrak{A}_{(V)}(\Lambda)$ denotes the local von Neumann algebra generated by all bounded functions of $\phi_{(V)}$ and $\pi_{(V)}$ smeared with real (periodic) test functions supported on $\Lambda$; see $[23,24]$.

We set

$$
\sim \Lambda \equiv\left\{x \mid x \in\left(\begin{array}{c}
\mathbb{R} \\
{[-V / 2, V / 2]}
\end{array}\right), x \notin \Lambda\right\}^{-},
$$

(depending on whether we are considering $(\phi, \mathscr{F})$ or $\left.\left(\phi_{V}, \mathscr{F}_{V}\right)\right) ;\{\cdots\}^{-}$denotes the closure of $\{\cdots\}$. If $\mathfrak{A}$ is an algebra of operators on a Hilbert space, $\mathfrak{A}^{\prime}$ denotes its commutant.

Lemma 1. The free, charged scalar fields $\phi, \phi_{V}$ satisfy duality, i.e.

$$
\mathfrak{U}(\Lambda)^{\prime}=\mathfrak{U}(\sim \Lambda), \mathfrak{A}_{V}(\Lambda)^{\prime}=\mathfrak{U}_{V}(\sim \Lambda) .
$$


Proof. For the free, neutral scalar field without cutoffs duality (27) has been proved in [34]. The method used in Osterwalder's proof [34] ( $\varphi-\pi$ expansions) works equally well for $\left(\phi_{V}, \pi_{V}, \mathscr{F}_{V}\right)$.

The theory of a free, charged scalar field is a tensor product of two identical copies of the theory of a free, neutral scalar field. In particular it follows from our definitions that

$$
\mathfrak{A}_{(V)}(\Lambda)=\mathfrak{U}_{(V)}^{1}(\Lambda) \bar{\otimes} \mathfrak{A}_{(V)}^{2}(\Lambda),
$$

where $\mathfrak{2}_{(V)}^{j}(\Lambda)$ is the local von Neumann algebra associated with $\phi_{(V), j}, \pi_{(V), j}$, $j=1,2$, and $\bar{\otimes}$ denotes tensor products of von Neumann algebras. Now

$$
\begin{aligned}
\mathfrak{A}_{(V)}(\Lambda)^{\prime} & =\left(\mathfrak{A}_{(V)}^{1}(\Lambda) \bar{\otimes} \mathfrak{H}_{(V)}^{2}(\Lambda)\right)^{\prime} \\
& =\mathfrak{U}_{(V)}^{1}(\Lambda)^{\prime} \bar{\otimes} \mathfrak{U}_{(V)}^{2}(\Lambda)^{\prime},
\end{aligned}
$$

by Tomita's commutant theorem [41]. Duality [34] yields

$$
\mathfrak{H}_{(V)}^{j}(\Lambda)^{\prime}=\mathfrak{H}_{(V)}^{j}(\sim \Lambda), \quad j=1,2,
$$

which completes the proof of (27).

Next we want to study the *-automorphism $\varrho_{\alpha}$ defined in (17) in some detail.

Let $\alpha$ be a real, continuously differentiable (for $V<\infty$ periodic)

function on the real line with $\partial_{x} \alpha \in L^{2}(\mathbb{R})$.

In the sense of quadratic forms we may define

$$
\begin{aligned}
L(\alpha) & \equiv \int d x\left[\phi_{1}(x) \pi_{2}(x)-\pi_{1}(x) \phi_{2}(x)\right] \alpha(x), \quad \text { and } \\
L_{V}(\alpha) & \equiv \int_{-V / 2}^{V / 2} d x\left[\phi_{V, 1}(x) \pi_{V, 2}(x)-\pi_{V, 1}(x) \phi_{V, 2}(x)\right] \alpha(x) .
\end{aligned}
$$

We set

$$
\Lambda_{\alpha} \equiv\{x \mid \alpha(x) \neq 2 m \pi, m \in \mathbb{Z}\}^{-} .
$$

Let $\mathscr{D}_{(V)}$ be the dense subspace of finite particle vectors in $\mathscr{F}_{(V)}$, i.e. vectors of the form

$$
\left\{f_{0}, f_{10}, f_{01}, \ldots, f_{j k}, \ldots\right\} \in \mathscr{F}_{(V)},
$$

with $f_{j k}=0$, for all $j>M, k>N$, for some finite integers $M$ and $N$.

Lemma 2. (1) Let $\alpha$ be as in (28) and $\alpha(x)=2 m \pi, m \in \mathbb{Z}$, for all $x \leqq x_{\alpha}$ with $x_{\alpha}$ some finite real number (depending on $\alpha$ ).

Then $L(\alpha)$ is essentially selfadjoint on $\mathscr{D}, e^{i L(\alpha)} \in \mathfrak{A}\left(\Lambda_{\alpha}\right)$, and $e^{i L(\alpha)}$ implements $\varrho_{\alpha}$.

(2) Let $\alpha$ be as in (28). Then $L_{V}(\alpha)$ is essentially selfadjoint on $\mathscr{D}_{V}, e^{i L_{V}(\alpha)} \in \mathfrak{U}_{V}\left(\Lambda_{\alpha}\right)$, and $e^{i L_{V}(\alpha)}$ implements $\varrho_{\alpha}$.

Proof. We denote the vector $\{1,0,0, \ldots\}$ - the bare vacuum - by $\Omega_{0}$ and set $\mu(k)=\sqrt{k^{2}+1}$. We first assume that $\alpha(x)=0$, for all $x \leqq x_{\alpha}$. Then

$$
\left\|L(\alpha) \Omega_{0}\right\|_{\mathscr{F}}^{2}=\int d k d p|\tilde{\alpha}(k+p)|^{2}(\mu(k)-\mu(p))^{2}(\mu(k) \mu(p))^{-1}
$$

is finite under our assumptions on $\alpha$ which imply that $|k| \tilde{\alpha}(k)$ is square integrable. 
It is then standard to show that $\mathscr{D}$ is a domain of analytic vectors for $L(\alpha)$ so that $L(\alpha) \uparrow \mathscr{D}$ is essentially selfadjoint by Nelson's theorem. It is easy to see that for $\alpha \equiv 2 m \pi, m \in \mathbb{Z}$,

$$
e^{i L(\alpha)}=e^{i 2 m \pi Q}=I \text {, }
$$

where $Q$ is the charge operator on $\mathscr{F}$. We conclude that

$$
e^{i L(\alpha+2 m \pi)} \equiv e^{i(L(\alpha)+2 m \pi Q)}=e^{i L(\alpha)},
$$

i.e. we can always choose $\alpha$ such that $\alpha(x)=0$, for $x \leqq x_{\alpha}$. One can show quite easily that

$$
e^{i L(\alpha)} e^{i \phi_{j}(f)} e^{-i L(\alpha)}=e^{i \varrho_{\alpha}\left(\phi_{j}\right)(f)} \equiv \varrho_{\alpha}\left(e^{i \phi_{j}(f)}\right),
$$

where $\varrho_{\alpha}\left(\phi_{j}\right)$ is defined by (17), plus identical equations for $e^{i \pi_{j}(f)}$; i.e. $e^{i L(\alpha)} \mathrm{im}$ plements $\varrho_{\alpha}$ unitarily on $\mathscr{F}$.

(On a space lattice Eq. (32) is a computation. To show it in the continuum limit one proves strong convergence of the lattice approximations of both sides in (32) following arguments of [27].)

Next let $\operatorname{supp} f \cong \sim \Lambda_{\alpha}$. It follows from (32) and (17) that

$$
e^{i L(\alpha)} e^{i \phi_{j}(f)} e^{-i L(\alpha)}=e^{i \phi_{j}(f)},
$$

and similarly for $e^{i \pi_{J}(f)}$. Since the operators

$$
\left\{e^{i \phi_{j}(f)} e^{i \pi_{j}(f)} \mid f \in \mathscr{S}_{\text {real }}(\mathbb{R}), \operatorname{supp} f \subseteq \sim \Lambda_{\alpha}, \quad j=1,2\right\}
$$

generate $\mathfrak{U}\left(\sim \Lambda_{\alpha}\right)$ we conclude that

$$
e^{i L(\alpha)} \in \mathfrak{U}\left(\sim \Lambda_{\alpha}\right)^{\prime}=\mathfrak{U}\left(\Lambda_{\alpha}\right), \quad \text { by Lemma } 1 .
$$

The proof of (2) is similar.

We remark that a direct proof of $e^{i L(\alpha)} \in \mathfrak{U}\left(\Lambda_{\alpha}\right)$ can be given, is however technically a little complicated in one space dimension.

Q.E.D.

Definition 2. (1) Let $\mathscr{H}$ be a Hilbert space, $A$ an operator on $\mathscr{H}$. The operator domain of $A$ is denoted by $D(A)$. We say that $A$ is symmetric (positive, selfadjoint ...) on $\mathscr{H}$ if $D(A)$ is dense in $\mathscr{H}$ and $A$ is symmetric (positive, selfadjoint ...) on $D(A)$. The quadratic form domain of $A$ is denoted by $Q(A)$. In our notations we do in general not distinguish between the operator $A$, its closure, the quadratic form determined by $A, \ldots$ The spectrum of $A$ is denoted by $\operatorname{spec} A$.

(2) We let $H_{O(V)}$ denote the free Hamiltonian of the charged, scalar field (in a periodic box $[-V / 2, V / 2])$ and define the Hamiltonian of the $(\boldsymbol{\phi} \cdot \boldsymbol{\phi})_{2}^{2}$-theory in a periodic box $[-V / 2, V / 2]$ as the selfadjoint operator determined by

$$
\begin{aligned}
H(l, V) \equiv H_{0 V} & +\lambda \int_{-l / 2}^{l / 2} d x:\left(\phi_{V} \cdot \phi_{V}\right)^{2}:(x) \\
& -\sigma \int_{-l / 2}^{l / 2} d x: \phi_{V, 1}^{2}:(x)-E(l, V),
\end{aligned}
$$

where $l \leqq V$ and $E(l, V)$ is the ground state energy (a unique selfadjoint operator can be associated with $H(l, V)$ e.g. by means of the Feynman-Kac formula [27]). 
For the Euclidean construction of the infinite volume limit $(\phi \cdot \phi)_{2}^{2}$-theory on the vacuum sector we also must consider the Hamiltonian defined by

$$
H(l, V, \mu) \equiv H(l, V)+\mu \int_{-l / 2}^{l / 2} d x \phi_{V, 1}(x)-E(l, V, \mu),
$$

with $\mu$ an arbitrary real number; see [20]. We set $H(l, \infty) \equiv H(l), H(l, \infty, \mu) \equiv H(l, \mu)$. For $\mu \neq 0$ the $\phi \rightarrow-\phi$ symmetry is explicitly broken. Hamiltonians similar to $H(l, V, \mu)$ have been discussed in [24] and from a Euclidean point of view in $[12,27]$. The results of these references apply to the present model after some straightforward changes.

Next we summarize some basic facts about the cutoff $(\phi \cdot \phi)_{2}^{2}$-theory.

Fact 1. For all $V \leqq \infty, l<\infty, \mu \in \mathbb{R}$ the Hamiltonian $H(l, V, \mu)$ is selfadjoint and bounded below by $0 ; 0$ is an eigenvalue of multiplicity 1 , i.e. $H(l, V, \mu)$ has a unique groundstate $\Omega_{l, V, \mu}$. We set

$$
\omega_{V, \mu}(\cdot)=\left\langle\Omega_{V, V, \mu}, \cdot \Omega_{V, V, \mu}\right\rangle_{\mathscr{F}_{V}},
$$

and remark that $\omega_{V, \mu}$ is a state on $\mathfrak{U}_{V}$.

Fact 2. Let $\Lambda$ be a finite union of compact intervals. We let $\mathfrak{\mathfrak { A }}_{(V)}(\Lambda)$ be the $C^{*}$-algebra generated by the operators

$$
\left\{e^{i \phi_{(V), j}(f)} e^{i \pi_{(V), j}(f)} \mid j=1,2, f \in \mathscr{S}_{\text {real }}(\mathbb{R}), \sup f \subseteq \Lambda\right\}
$$

and define $\varrho_{V}$ to be the *-isomorphism $\mathfrak{\mathfrak { U }}(\Lambda) \rightarrow \mathfrak{\mathfrak { U }}_{V}(\Lambda)$ defined by

$$
\varrho_{V}\left(\phi_{j}(f)\right)=\phi_{V, j}(f), \varrho_{V}\left(\pi_{j}(f)\right)=\pi_{V, j}(f) .
$$

If $\mathscr{F}_{V}$ is embedded in $\mathscr{F}$ as a subspace in the usual way [24] one can easily check that

$$
\text { s- } \lim _{V \rightarrow \infty} \varrho_{V}(A)=A, \quad \text { for all } A \in \mathfrak{\mathfrak { I }}(\Lambda) ;
$$

see [24] for precise definitions and proofs.

Fact 3. (Convergence of the periodic box cutoff [24])

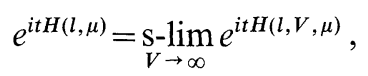

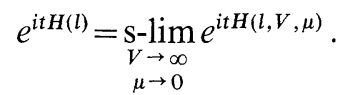

Fact 4. (Finite propagation speed, [24].) Given $\Lambda$ we define $\Lambda_{t} \equiv\{x \mid \operatorname{dist}(x, \Lambda) \leqq$ $|t|\}$.

For $A \in \mathfrak{U}_{V}(\Lambda)$ and arbitrary $\mu$

$$
e^{i t H(l, V, \mu)} A e^{-i t H(l, V, \mu)} \in \mathfrak{A}_{V}\left(\Lambda_{t}\right) .
$$

If $\Lambda_{t} \subset[-l / 2, l / 2]$ then

$$
\left.e^{i t H(l, V, \mu)} A e^{-i t H(l, V, \mu)}\right)=e^{i t H(V, V, \mu)} A e^{-i t H(V, V, \mu)}
$$

is independent of $l$. 
Fact 5. (Construction of the time-translation automorphism [24].) If $\Lambda_{t} \mathrm{C}$ $[-l / 2, l / 2]$ and $A \in \mathfrak{Q} \mathfrak{i}(\Lambda)$ then

$$
\begin{aligned}
& \underset{V \rightarrow \infty}{S-l \lim e^{i t H(l, V, \mu)}} \varrho_{V}(A) e^{-i t H(l, V, \mu)} \\
& =e^{i t H(l, \mu)} A e^{-i t H(l, \mu)} \equiv \tau_{t}^{\mu}(A) ;
\end{aligned}
$$

$\tau_{t}^{\mu}(A)$ is contained in $\mathfrak{H}\left(\Lambda_{t}\right)$ and is independent of $l ; \tau_{t}^{\mu}$ is the infinite volume timetranslation automorphism of $\mathfrak{A}$ constructed by Glimm and Jaffe [22, 24]. Notice that $\tau_{t}^{\mu}$ has unit propagation speed.

Fact 6. For $A \in \bigcup_{\{\Lambda\}} \mathfrak{A}(\Lambda)$

$$
\mathrm{s}_{\mu \rightarrow 0} \lim _{t}^{\mu}(A)=\tau_{t}^{\mu=0}(A) \equiv \tau_{t}(A) .
$$

This follows from Fact 3, (36).

Definition 3. For $\alpha$ as in Lemma 2, (2) we define

$$
e^{i t H(\alpha \mid l, V, \mu)} \equiv e^{i L_{V}(\alpha)} e^{i t H(l, V, \mu)} e^{-i L_{V}(\alpha)},
$$

and for $\alpha$ as in Lemma 2, (1) we set

$$
e^{i t H(\alpha \mid l, \mu)} \equiv e^{i L(\alpha)} e^{i t H(l, \mu)} e^{-i L(\alpha)} .
$$

The following "propagators" play an important role:

$$
\begin{aligned}
V_{t}(\alpha \mid l, V, \mu) & \equiv e^{i t H(\alpha \mid l, V, \mu)} e^{-i t H(l, V, \mu)}, \\
V_{t}(\alpha \mid l, \mu) & \equiv e^{i t H(\alpha \mid l, \mu)} e^{-i t H(l, \mu)} .
\end{aligned}
$$

Fact 7. For $\alpha$ as in (28) and $\alpha(x)=2 m \pi$, for some $m \in \mathbb{Z}$, all $x \leqq \underline{x}_{\alpha}, x \geqq \bar{x}_{\alpha}$, with $\underline{x}_{\alpha}<\bar{x}_{\alpha}$

$$
\mathrm{S}_{V \rightarrow \infty} \lim ^{i L_{V}(\alpha)}=e^{i L(\alpha)} .
$$

(Choose $\Psi$ in $\mathscr{D}$. Then $\operatorname{s-lim}_{V \rightarrow \infty} L_{V}(\alpha) \Psi=L(\alpha) \Psi$ and hence, using Duhamel's formula, $e^{i L_{V}(\alpha)} \Psi \rightarrow e^{i L(\alpha)} \Psi$, strongly. Since $\mathscr{D}$ is dense in $\mathscr{F}$ the proof is complete.) Combining this with Fact 3 and the unitarity of $e^{i L_{V}(\alpha)}$ and $e^{i t H(l, V, \mu)}$ we obtain

$$
\begin{aligned}
& \underset{V \rightarrow \infty}{s-\lim _{t}} V_{t}(\alpha \mid l, V, \mu)=V_{t}(\alpha \mid l, \mu), \\
& \underset{\substack{V \rightarrow \infty \\
\mu \rightarrow 0}}{\mathrm{~s}-\lim _{t}} V_{t}(\alpha \mid l, V, \mu)=V_{t}(\alpha \mid l, 0)=V_{t}(\alpha \mid l) .
\end{aligned}
$$

Remark. There is no explicit expression for $H(\alpha \mid l, V, \mu)$. The domains $D(H(l, V, \mu))$ and $D(H(\alpha \mid l, V, \mu))$ are disjoint. [The formal expression for $H(\alpha \mid l, V, \mu)-$ $H(l, V, \mu)$ is ultraviolet divergent.]

However, if one considers the $(\phi \cdot \phi)_{2}^{2}$-theory on a space lattice with lattice constant $\delta$ and lets $H(l, \delta, \mu), H(\alpha \mid l, \delta, \mu)$ be the corresponding lattice Hamiltonians one can derive an explicit expression for $H(\alpha \mid l, \delta, \mu): H(\alpha \mid l, \delta, \mu)=H(l, \delta, \mu)$ $\delta H(\alpha \mid l, \delta, \mu)$, where $\delta H(\alpha \mid l, \delta, \mu) \equiv \delta H(\alpha \mid \delta, \mu)$ is independent of $l$ if $\Lambda_{\alpha} \subset[-l / 2, l / 2]$; $\delta H(\alpha \mid \delta, \mu)$ is a tiny form perturbation of $H(l, \delta, \mu)$, uniformly in $l<\infty$. [An explicit computation of $\delta H(\alpha \mid \delta, \mu)$ is left to the reader.] As we let $\delta$ tend to 0 the perturba- 
tion $\delta H(\alpha \mid \delta, \mu)$ develops ultraviolet singularities and the domains of $H(l, \delta, \mu)$ and $H(\alpha \mid l, \delta, \mu)$ separate in the limit $\delta=0$.

It is easy to check that $\left\{e^{-t H(\alpha \mid l, \mu)} / t \geqq 0\right\}$ is a positivity preserving contraction semigroup on $L^{2}\left(\mathscr{S}_{\text {real }}^{\prime}(\mathbb{R})^{\times 2}, d \mu\right) \cong \mathscr{F}$ which can be extended to $L^{p}\left(\mathscr{S}_{\text {real }}^{\prime}(\mathbb{R})^{\times 2}, d \mu\right)$, $1<p<2$. [Here $d \mu$ is the usual Gaussian measure on the $Q$-space $\mathscr{S}_{\text {real }}^{\prime}(\mathbb{R})^{\times 2}$ of the charged scalar time 0 -field.] Therefore it ought to be possible to replace our algebraic proof of Theorem 3 started in Lemmata 1 and 2 and pursued below by a Euclidean proof using path space techniques - as it is possible in the case of the $\cos \varepsilon \phi_{2}$-theory.

After this break let us again do some work. The following lemma is the basic ingredient for the proof of Theorem 3.

Lemma 3. (1) Let $\alpha$ be as in Lemma 2, (2) and suppose that $\Lambda_{\alpha, s} \subseteq[-l / 2, l / 2]$, for some $s>0$. Then for all $|t|<s, V_{t}(\alpha \mid l, V, \mu) \equiv V_{t}(\alpha \mid V, \mu)$ is contained in $\mathfrak{U}_{V}\left(\Lambda_{\alpha, t}\right)$ and is independent of $l$; it is strongly continuous in $t$.

(2) Let $\alpha$ be as in Lemma 2, (1) and assume that $\Lambda_{\alpha, s} \subseteq[-l / 2, l / 2]$, for some $s>0$. Then for all $|t|<s, V_{t}(\alpha \mid \mu)$ is contained in $\mathfrak{U}\left(\Lambda_{\alpha, t}\right)$ and is independent of $l$. It is strongly continuous in $t$.

(3) Let $\mu=0$ and define

$$
\overline{\Lambda_{\alpha}} \equiv\{x \mid \alpha(x) \neq m \pi, m \in \mathbb{Z}\} \text {. }
$$

Then $V_{t}(\alpha \mid l)$ is contained in $\mathfrak{A}\left(\bar{\Lambda}_{\alpha, t}\right)$. If $\bar{\Lambda}_{\alpha, s} \cong[-l / 2, l / 2]$ then for all $|t|<s, V_{t}(\alpha \mid l) \equiv$ $V_{t}(\alpha)$ is independent of $l$. $V_{t}(\alpha)$ is strongly continuous in $t$.

(4) Let $\mu=0, \alpha=\alpha_{1}+\alpha_{2}$ with $\alpha_{1}, \alpha_{2}$ such as in Lemma 2, (1) and assume that $\bar{\Lambda}_{\alpha_{1}, t} \cap \bar{\Lambda}_{\alpha_{2}, t}=\emptyset$.

Then

$$
V_{t}(\alpha \mid l)=V_{t}\left(\alpha_{1} \mid l\right) V_{t}\left(\alpha_{2} \mid l\right) \text {, }
$$

with

$V_{t}\left(\alpha_{j} \mid l\right) \in \mathfrak{X}\left(\bar{\Lambda}_{\alpha_{j}, t}\right), \quad j=1,2$.

Proof. (1) By Lemma 2, (2) $e^{ \pm i L_{V}(\alpha)} \in \mathfrak{A}_{V}\left(\Lambda_{\alpha}\right)$. Thus

$$
e^{i t H(l, V, \mu)} e^{-i L_{V}(\alpha)} e^{-i t H(l, V, \mu)} \in \mathfrak{U}_{V}\left(\Lambda_{\alpha, t}\right),
$$

by Fact 4 . Therefore $V_{t}(\alpha \mid l, V, \mu) \in \mathfrak{U}_{V}\left(\Lambda_{\alpha, t}\right)$. If $\Lambda_{\alpha, t} \subset[-l / 2, l / 2] \quad$ then $e^{i t H(l, V, \mu)} e^{i L_{V}(\alpha)} e^{-i t H(l, V, \mu)}=e^{i t H(V, V, \mu)} e^{-i L_{V}(\alpha)} e^{-i t H(V, V, \mu)}$ is independent of $l$, by Fact 4, and hence so is $V_{t}(\alpha \mid l, V, \mu)$. Finally $V_{t}(\alpha \mid l, V, \mu)$ is strongly continuous in $t$, since $\left\{e^{i t H(l, V, \mu)} \mid t \in \mathbb{R}\right\}$ is a strongly continuous unitary group and $e^{i L_{V}(\alpha)}$ is unitary on $\mathscr{F}_{V}$. The proof of (2) is identical (the only difference being that $\alpha$ need no longer be periodic).

(3) Let $\varrho_{\pi}$ be the $*$-automorphism on $\mathfrak{A}$ defined by

$$
\left.\begin{array}{l}
\varrho_{\pi}\left(e^{i \phi_{j}(f)}\right)=e^{-i \phi_{j}(f)}, \\
\left.\varrho_{\pi}\left(e^{i \pi_{j}(f)}\right)=e^{-i \pi_{j}(f)}, \quad j=1,2 .\right\}
\end{array}\right\}
$$

Clearly $\varrho_{\pi}^{2}=I$.

If $A \in \mathfrak{U}(\Lambda)$ and $\alpha(x)=(2 m+1) \pi$, for all $x \in \Lambda$, then by Lemma $2,(1)$

$$
e^{ \pm i L(\alpha)} A e^{\bar{\mp} i L(\alpha)}=\varrho_{ \pm \alpha}(A)=\varrho_{\pi}(A) \text {. }
$$


For $\mu=0$ and all $A \in \mathfrak{A}$

$$
e^{i t H(l)} \varrho_{\pi}(A) e^{-i t H(l)}=\varrho_{\pi}\left(e^{i t H(l)} A e^{i t H(l)}\right),
$$

by the $\phi \rightarrow-\phi$ symmetry of $H(l)$. (The operators $H_{0}$ and $H(l)-H_{0}$ are obviously invariant under $\phi \mapsto-\phi ;$; (39) follows therefore from the Trotter product formula.)

Now let $A \in \mathfrak{A}\left(\sim \bar{\Lambda}_{\alpha, t}\right)$. Then

$e^{-i t H(l)} A e^{i t H(l)} \in \mathfrak{A}\left(\sim \bar{\Lambda}_{\alpha}\right)$,

by Fact 5 (unit propagation speed).

Thus, using (37)-(39)

$$
\begin{aligned}
& V_{t}(\alpha \mid l) A V_{t}^{*}(\alpha \mid l) \\
& =e^{i L(\alpha)} e^{i t H(l)} e^{-i L(\alpha)} e^{-i t H(l)} A e^{i t H(l)} e^{i L(\alpha)} e^{-i t H(l)} e^{-i L(\alpha)} \\
& =e^{i L(\alpha)} e^{i t H(l)} \varrho_{\pi}\left(e^{-i t H(l)} A e^{i t H(l)}\right) e^{-i t H(l)} e^{-i L(\alpha)} \\
& =e^{i L(\alpha)} \varrho_{\pi}(A) e^{-i L(\alpha)}=\varrho_{\pi}\left(\varrho_{\pi}(A)\right)=A .
\end{aligned}
$$

Therefore $V_{t}(\alpha \mid l) \in \mathfrak{H}\left(\sim \overline{\Lambda_{\alpha, t}}\right)^{\prime}=\mathfrak{A}\left(\overline{\Lambda_{\alpha, t}}\right)$, by duality (Lemma 1). Next let $W(l) \equiv$ $H(l)-H_{0}$ be the spatially cutoff $(\boldsymbol{\phi} \cdot \boldsymbol{\phi})_{2}^{2}$-interaction. We set

$$
W(l, \tau) \equiv e^{-i \tau H_{0}} W(l) e^{i \tau H_{0}} .
$$

By the Trotter product formula - see $[22,24]-$

$$
\begin{aligned}
& e^{i L(\alpha)} e^{i t H(l)} e^{-i L(\alpha)} e^{-i t H(l)} \\
& =\underset{n \rightarrow \infty}{\operatorname{sim}} e^{i L(\alpha)} e^{i t H_{0}} \prod_{j=0}^{n-1} e^{i \frac{t}{n} W\left(l,\left(1-\frac{j}{n}\right) t\right)} \\
& \quad \times e^{-i L(\alpha)} \prod_{j=1}^{n} e^{-i \frac{t}{n} W\left(l, \frac{j}{n} t\right)} e^{-i t H_{0}} .
\end{aligned}
$$

We let $a^{\prime}>a$ and define

$$
W\left(a^{\prime}, a, \tau\right) \equiv W\left(a^{\prime}, \tau\right)-W(a, \tau) .
$$

For all $a^{\prime}, a$ and $\tau, e^{i W\left(a^{\prime}, a, \tau\right)}$ is

invariant under the substitution $\phi \rightarrow-\phi$.

$$
\begin{aligned}
& \text { Now for } \bar{\Lambda}_{\alpha, \tau} \subseteq\left[-\frac{a}{2}, \frac{a}{2}\right] \\
& e^{i W\left(a^{\prime}, a, \tau\right)} \in \mathfrak{U}\left(\sim \bar{\Lambda}_{\alpha, \tau}\right)
\end{aligned}
$$

and therefore it commutes with $e^{-i L(\alpha)}$ by Lemma 2, (1), Definition (17) of $\varrho_{\alpha}$ and (41).

Finally

$$
e^{i W\left(a^{\prime}+\tau, a+\tau, \tau\right)} \quad \text { commutes with } e^{i W(a)} .
$$

Let $a_{0}=\min \left\{a \mid \bar{\Lambda}_{\alpha} \subseteq\left[-\frac{a}{2}, \frac{a}{2}\right]\right\}$. Since $|t|<s$ and $\bar{\Lambda}_{\alpha, s} \subseteq\left[-\frac{l}{2}, \frac{l}{2}\right], a_{0}+|t|<l$. 
Thus by (41) and (42)

$$
\begin{aligned}
& e^{i L(\alpha)} e^{i t H_{0}} \prod_{j=0}^{n-1} e^{i \frac{t}{n} W\left(l,\left(1-\frac{j}{n}\right) t\right)} e^{-i L(\alpha)} \\
& \times \prod_{j=1}^{n} e^{-i \frac{t}{n} W\left(l, \frac{j}{n} t\right)} e^{-i t H_{0}} \\
& =e^{i L(\alpha)} e^{i t H_{0}} \prod_{j=0}^{n-1} e^{i \frac{t}{n} W\left(a_{0}+\left(1-\frac{j}{n}\right) t,\left(1-\frac{j}{n}\right) t\right)} e^{-i L(\alpha)} \\
& \times \prod_{j=1}^{n} e^{-i \frac{t}{n} W\left(a_{0}+\frac{j}{n} t, \frac{j}{n} t\right)} e^{-i t H_{0}}
\end{aligned}
$$

The r.h.s. of Eq. (43) is obviously independent of $l$. By Eq. (40) it converges strongly as $n \rightarrow \infty$. The limit is denoted by $V_{t}(\alpha) ; V_{t}(\alpha)$ is strongly continuous in $t$ by (40) and Lemma 3, (2). This completes the proof of (3).

(4) By Lemma 2, (1), Definition (17) of $\varrho_{\alpha}$ and the fact that $S O(2)$ is an abelian group we have

$$
e^{i L\left(\alpha_{1}+\alpha_{2}\right)} A e^{-i L\left(\alpha_{1}+\alpha_{2}\right)}=e^{i L\left(\alpha_{1}\right)} e^{i L\left(\alpha_{2}\right)} A e^{-i L\left(\alpha_{2}\right)} e^{-i L\left(\alpha_{1}\right)},
$$

for all $A \in \mathfrak{U}$. Since $\mathfrak{U}$ acts irreducibly on $\mathscr{F}$, this equation is true for all bounded operators on $\mathscr{F}$. Thus

$$
\begin{aligned}
e^{i L\left(\alpha_{1}+\alpha_{2}\right)} e^{i t H(l)} e^{-i L\left(\alpha_{1}+\alpha_{2}\right)} e^{-i t H(l)} \\
=e^{i L\left(\alpha_{1}\right)}\left(e^{i L\left(\alpha_{2}\right)} e^{i t H(l)} e^{-i L\left(\alpha_{2}\right)} e^{-i t H(l)}\right) \\
\quad \times\left(e^{i t H(l)} e^{-i L\left(\alpha_{1}\right)} e^{-i t H(l)} e^{i L\left(\alpha_{1}\right)}\right) e^{-i L\left(\alpha_{1}\right)} \\
=e^{i L\left(\alpha_{1}\right)} V_{t}\left(\alpha_{2} \mid l\right) V_{t}^{*}\left(-\alpha_{1} \mid l\right) e^{-i L\left(\alpha_{1}\right)} \\
=e^{i L\left(\alpha_{1}\right)} V_{t}^{*}\left(-\alpha_{1} \mid l\right) V_{t}\left(\alpha_{2} \mid l\right) e^{-i L\left(\alpha_{1}\right)},
\end{aligned}
$$

by Lemma 3, (3) and the fact that

$$
\mathfrak{U}\left(\bar{\Lambda}_{\alpha_{1}, t}\right) \subset \mathfrak{U}\left(\bar{\Lambda}_{\alpha_{2}, t}\right)^{\prime}, \quad \text { for } \quad \bar{\Lambda}_{\alpha_{1}, t} \cap \bar{\Lambda}_{\alpha_{2}, t}=\emptyset \text {. }
$$

Clearly $e^{i L\left(\alpha_{1}\right)} V_{t}^{*}\left(-\alpha_{1} \mid l\right)=V_{t}\left(\alpha_{1} \mid l\right) e^{i L\left(\alpha_{1}\right)}$.

Since $\bar{\Lambda}_{\alpha_{1}, t} \cap \bar{\Lambda}_{\alpha_{2}, t}=\emptyset$,

$$
e^{i L\left(\alpha_{1}\right)} V_{t}\left(\alpha_{2} \mid l\right) e^{-i L\left(\alpha_{1}\right)}=\left\{\begin{array}{l}
\varrho_{\pi}\left(V_{t}\left(\alpha_{2} \mid l\right)\right), \\
V_{t}\left(\alpha_{2} \mid l\right),
\end{array}\right.
$$

depending on whether $\alpha_{1}(x)$ is an odd or an even multiple of $\pi$ for $x \in \bar{\Lambda}_{\alpha_{2}, t}$. As a consequence of the $\phi \rightarrow-\phi$ invariance of $H(l)$ we have

$$
\varrho_{\pi}\left(V_{t}\left(\alpha_{2} \mid l\right)\right)=V_{t}\left(\alpha_{2} \mid l\right)
$$

as one easily checks.

Therefore

$$
\begin{aligned}
& e^{i L\left(\alpha_{1}+\alpha_{2}\right)} e^{i t H(l)} e^{-i L\left(\alpha_{1}+\alpha_{2}\right)} e^{-i t H(l)} \\
& =V_{t}\left(\alpha_{1} \mid l\right) e^{i L\left(\alpha_{1}\right)} V_{t}\left(\alpha_{2} \mid l\right) e^{-i L\left(\alpha_{1}\right)} \\
& =V_{t}\left(\alpha_{1} \mid l\right) V_{t}\left(\alpha_{2} \mid l\right)
\end{aligned}
$$

which completes the proof of (4). 
Definition 4 . We abbreviate $(l, V, \mu)$ by $\gamma$. A point $(t, x) \in \mathbb{R}^{2}$ is denoted by $\xi$, and $T_{\xi}^{\gamma} \equiv e^{i(t H(\gamma)-x P(\gamma))}$,

where $P(\gamma) \equiv P(V)$ is the momentum operator on $\mathscr{F}_{V}(=\mathscr{F}$, for $V=\infty)$. We set

$$
\begin{aligned}
\tau_{\xi}^{\gamma}(A) & \equiv T_{\xi}^{\gamma} A\left(T_{\xi}^{\gamma}\right)^{*}, A \in \mathfrak{U}_{V} \\
\tau_{t}^{\gamma} & \equiv \tau_{(t, 0)}^{\gamma} \\
L(\alpha \mid \gamma) & \equiv L_{V}(\alpha)(=L(\alpha) \quad \text { for } \quad V=\infty),
\end{aligned}
$$

and

$$
\begin{aligned}
& V_{\xi}(\alpha \mid \gamma) \equiv e^{i L(\alpha \mid \gamma)} e^{i(t H(\gamma)-x P(\gamma))} \\
& \times e^{-i L(\alpha \mid \gamma)} e^{-i(t H(\gamma)-x P(\gamma))} \\
&=V_{t}(\alpha \mid \gamma) \tau_{t}^{\gamma}\left(e^{i L\left(\alpha-\alpha_{x} \mid \gamma\right)}\right)
\end{aligned}
$$

where $\alpha_{x}(y) \equiv \alpha(y-x)$.

Notice that $\tau_{t}^{\gamma}\left(e^{i L\left(\alpha-\alpha_{x} \mid \gamma\right)}\right) \in \mathfrak{U}_{V}\left(\Lambda_{\alpha_{x}-\alpha, t}\right)$.

Lemma 4. For all $l<\infty, V \leqq \infty$ and arbitrary $\mu$

(1) $V_{\xi_{1}}(\alpha \mid \gamma) T_{\xi_{1}}^{\gamma} V_{\xi_{2}}(\alpha \mid \gamma) T_{\xi_{2}}^{\gamma}$

$$
\begin{aligned}
& =V_{\xi_{1}}(\alpha \mid \gamma) \tau_{\xi_{1}}^{\gamma}\left(V_{\xi_{2}}(\alpha \mid \gamma)\right) T_{\xi_{1}+\xi_{2}}^{\gamma} \\
& =V_{\xi_{1}+\xi_{2}}(\alpha \mid \gamma) T_{\xi_{1}+\xi_{2}}^{\gamma} .
\end{aligned}
$$

(2) $V_{\xi}^{*}(\alpha \mid \gamma) \varrho_{\alpha}(A) V_{\xi}(\alpha \mid \gamma)$

$$
=\tau_{\xi}^{\gamma}\left(\varrho_{\alpha}\left(\tau_{-\xi}^{\gamma}(A)\right)\right) \text {. }
$$

Proof. This lemma is an immediate consequence of Definitions 3 and 4; e.g.

$$
\begin{aligned}
V_{\xi}^{*}(\alpha \mid \gamma) \varrho_{\alpha}(A) V_{\xi}(\alpha \mid \gamma)= & T_{\xi}^{\gamma} e^{i L(\alpha)} T_{-\xi}^{\gamma} e^{-i L(\alpha)} \\
& \times \varrho_{\alpha}(A) e^{i L(\alpha)} T_{\xi}^{\gamma} e^{-i L(\alpha)} T_{-\xi}^{\gamma} \\
= & T_{\xi}^{\gamma} e^{i L(\alpha)} \tau_{-\xi}^{\gamma}(A) e^{-i L(\alpha)} T_{-\xi}^{\gamma} \\
= & \tau_{\xi}^{\gamma}\left(\varrho_{\alpha}\left(\tau_{-\xi}^{\gamma}(A)\right)\right) .
\end{aligned}
$$

Next we construct the infinite volume limit $V \rightarrow \infty$ and then pass to the limit $\mu=0$.

Definition 5. Let $\alpha^{+}$satisfy the soliton-condition (21), i.e. $\alpha^{+}$is continuously differentiable and

$$
\lim _{x \rightarrow-\infty} \alpha^{+}(x)=0, \lim _{x \rightarrow+\infty} \alpha^{+}(x)=\pi, \operatorname{supp}\left(\partial_{x} \alpha^{+}\right) \text {compact . }
$$

We define $\alpha^{-}(x) \equiv \alpha^{+}(-x) ; \alpha^{e}(x) \equiv \alpha^{ \pm}(x)$, for $e= \pm 1$,

$$
\alpha^{e, n}(x) \equiv \alpha^{e}(x)-\alpha^{e}(x-n),
$$

where $n$ is some integer.

The cutoff vacuum states $\omega_{V, \mu}$ have been defined in Fact 1, (34), the isomorphism $\varrho_{V}: \mathfrak{U} \rightarrow \mathfrak{U}_{V}$ in Fact 2. 
Lemma 5. For $\mu \neq 0$

(1) $\lim _{V \rightarrow \infty} \omega_{V, \mu}\left(\varrho_{V}(A) V_{\xi_{1}}\left(\alpha^{e, n} \mid \gamma\right) \tau_{\xi_{1}}^{\gamma}\left(V_{\xi_{2}}\left(\alpha^{e, n} \mid \gamma\right)\right) \tau_{\xi_{1}+\xi_{2}}^{\gamma}\left(\varrho_{V}(B)\right)\right)$

$=\omega_{\mu}\left(A V_{\xi_{1}}\left(\alpha^{e, n} \mid \mu\right) T_{\xi_{1}}^{\mu} V_{\xi_{2}}\left(\alpha^{e, n} \mid \mu\right) T_{\xi_{2}}^{\mu} B\right)$

$=\omega_{\mu}\left(A V_{\xi_{1}+\xi_{2}}\left(\alpha^{e, n} \mid \mu\right) T_{\xi_{1}+\xi_{2}}^{\mu} B\right)$,

for all $A$ and $B$ in $\mathfrak{\mathfrak { U }}$.

(2) $\lim _{V \rightarrow \infty} \omega_{V, \mu}\left(V_{\xi}^{*}\left(\alpha^{e, n} \mid \gamma\right) \varrho_{\alpha^{e, n}}\left(\varrho_{V}(A)\right) V_{\xi}\left(\alpha^{e, n} \mid \gamma\right)\right)$

$=\omega_{\mu}\left(V_{\xi}^{*}\left(\alpha^{e, n} \mid \mu\right) \varrho_{\alpha^{e, n}}(A) V_{\xi}\left(\alpha^{e, n} \mid \mu\right)\right)$

$=\omega_{\mu}\left(\varrho_{\alpha^{e, n}}\left(\tau_{-\xi}^{\mu}(A)\right)\right)$.

(3) There exists a decreasing sequence $\left\{\mu_{l}\right\}_{l=1}^{\infty}$ converging to 0 such that

$$
\begin{aligned}
& \lim _{l \rightarrow \infty} \omega_{ \pm \mu_{l}}\left(A V_{\xi_{1}}\left(\alpha^{e, n} \mid \pm \mu_{l}\right) T_{\xi_{1}}^{ \pm \mu_{l}} V_{\xi_{2}}\left(\alpha^{e, n} \mid \pm \mu_{l}\right) T_{\xi_{2}}^{ \pm \mu_{l}} B\right) \\
& =\omega_{ \pm}\left(A V_{\xi_{1}}\left(\alpha^{e, n)} T_{\xi_{1}} V_{\xi_{2}}\left(\alpha^{e, n}\right) T_{\xi_{2}} B\right)\right. \\
& =\omega_{ \pm}\left(A V_{\xi_{1}+\xi_{2}}\left(\alpha^{e, n}\right) T_{\xi_{1}+\xi_{2}} B\right)
\end{aligned}
$$

(4) $\lim _{l \rightarrow \infty} \omega_{ \pm \mu_{l}}\left(V_{\xi}^{*}\left(\alpha^{e, n} \mid \pm \mu_{l}\right) \varrho_{\alpha^{e, n}}(A) V_{\xi}\left(\alpha^{e, n} \mid \pm \mu_{l}\right)\right)$

$=\omega_{ \pm}\left(V_{\xi}^{*}\left(\alpha^{e, n}\right) \varrho_{\alpha^{e, n}}(A) V_{\xi}\left(\alpha^{e, n}\right)\right)$

$=\omega_{ \pm}\left(\varrho_{\alpha^{e, n}}\left(\tau_{-\xi}(A)\right)\right)$.

(5) For $\phi=\left(\phi_{1}, \ldots, \phi_{n}\right)$ with $n=1$ or 2 the limits in (3) and (4) are independent of the choice of the sequence $\left\{\mu_{l}\right\}_{l=1}^{\infty}$.

Proof. This lemma looks complicated but is easy. We use the locally Fock property - more precisely the local norm-compactness of the family $\left\{\omega_{V, \pm \mu} \mid V \leqq \infty\right.$, $\mu \geqq 0\}$ of states $[23,24]$ - to transfer the relations of Lemma 4 to the limits $V=\infty$, $\mu=0$.

(1) It suffices to prove (1) for $A$ and $B$ in $\mathfrak{\mathfrak { U }}(\Lambda)$, for some arbitrary, but bounded interval $\Lambda$. The convergence of $\varrho_{V}$ to the identity, as $V \rightarrow \infty$, on such operators follows from Fact 2, (35). The convergence of the vacuum states $\omega_{V, \mu}$, as $V \rightarrow \infty$, is a consequence of [20] [see also Theorem 1, (b) and (c)] and [24, 26]. By Lemma 4, (1)

$$
\begin{aligned}
& V_{\xi_{1}}\left(\alpha^{e, n} \mid \gamma\right) \tau_{\xi_{1}}^{\gamma}\left(V_{\xi_{2}}\left(\alpha^{e, n} \mid \gamma\right)\right) \tau_{\xi_{1}+\xi_{2}}^{\gamma}\left(\varrho_{V}(B)\right) \\
& =V_{\xi_{1}+\xi_{2}}\left(\alpha^{e, n} \mid \gamma\right) \tau_{\xi_{1}+\xi_{2}}^{\gamma}\left(\varrho_{V}(B)\right) .
\end{aligned}
$$

Next we use Fact 2, (35), Facts 3 and 4, Fact 7, Lemma 3, (1) and (2), and (44) and (45) to conclude that

$$
\begin{aligned}
& \underset{V \rightarrow \infty}{\operatorname{s}-\lim } \varrho_{V}(A) V_{\xi}\left(\alpha^{e, n} \mid \gamma\right) \tau_{\xi_{1}}^{\gamma}\left(V_{\xi_{2}}\left(\alpha^{e, n} \mid \gamma\right)\right) \tau_{\xi_{1}+\xi_{2}}^{\gamma}\left(\varrho_{V}(B)\right) \\
& =A V_{\xi_{1}}\left(\alpha^{e, n} \mid \mu\right) \tau_{\xi_{1}}^{\mu}\left(V_{\xi_{2}}\left(\alpha^{e, n} \mid \mu\right)\right) \tau_{\xi_{1}+\xi_{2}}^{\mu}(B),
\end{aligned}
$$

and

$$
\begin{aligned}
& \underset{V \rightarrow \infty}{\mathrm{s}-\lim _{V} \varrho_{V}(A) V_{\xi_{1}+\xi_{2}}\left(\alpha^{e, n} \mid \gamma\right) \tau_{\xi_{1}}^{\gamma}+\xi_{2}}\left(\varrho_{V}(B)\right) \\
& =A V_{\xi_{1}+\xi_{2}}\left(\alpha^{e, n} \mid \mu\right) \tau_{\xi_{1}+\xi_{2}}^{\mu}(B) .
\end{aligned}
$$

Lemma 5, (1) now follows from (47) and Theorem 3.1 of Ref. [24]. 
(2) By Lemma 4, (2)

$$
\begin{aligned}
& V_{\xi}^{*}\left(\alpha^{e, n} \mid \gamma\right) \varrho_{\alpha^{e, n}}\left(\varrho_{V}(A)\right) V_{\xi}\left(\alpha^{e, n} \mid \gamma\right) \\
& =\tau_{\xi}^{\gamma}\left(\varrho_{\alpha}\left[\tau_{-\xi}^{\gamma}\left(\varrho_{V}(A)\right)\right]\right) .
\end{aligned}
$$

By Fact 2, (35), Facts 3 and 4, Fact 7, Lemma 3, (1) and (2), and (44) and (45) the left and the r.h.s. of (48) converge strongly on $\mathscr{F}$ to

$$
\begin{aligned}
& V_{\xi}^{*}\left(\alpha^{e, n} \mid \mu\right) \varrho_{\alpha^{e, n}}(A) V_{\xi}\left(\alpha^{e, n} \mid \mu\right), \\
& \tau_{\xi}^{\mu}\left(\varrho_{\alpha}\left[\tau_{-\xi}^{\mu}(A)\right]\right), \text { respectively, as } V \rightarrow \infty .
\end{aligned}
$$

Application of [24, Theorem 3.1] and the convergence of the states $\omega_{V, \mu}$ [20] completes the proof of (2). The proofs of (3) and (4) are similar to the ones of (1), (2), resp.: We combine Facts 3-7 and Lemma 5, (1) and (2), with a straightforward extension of [24, Theorem 3.1, (3.5)].

(5) This follows from correlation inequalities of [27] for the case $n=1$ and of [11] for the case $n=2$. Some details are given in [20]. For $n \geqq 3$ the relevant correlation inequalities (yielding uniqueness of the limit $\mu \rightarrow 0$ ) are not known.

Q.E.D.

Remark. The theory reconstructed from the limiting states $\omega_{ \pm}$satisfies all Wightman axioms with the possible exception of the cluster properties; see Theorem 1, (b) and (c), and [20].

Next we want to let $n$ tend to $\infty$. The states $\omega_{ \pm}{ }^{\circ} \varrho_{\alpha^{e, n}}$ represent "soliton-antisoliton states". The limit $n \rightarrow \infty$ can be interpreted as "sending the soliton (or anti-soliton) to behind the moon". It has not been proved that the vacuum states $\omega_{ \pm}$have spatial cluster decomposition properties - i.e. are pure states - and thus the limit $n \rightarrow \infty$ might not be meaningful. Therefore we must first decompose the vacuum states $\omega_{ \pm}$into pure phase states which do have spatial cluster properties.

Lemma 6. There exists a compact Hausdorff space $X$ (points in $X$ are interpreted as pure phases), and a regular Borel probability measure $v_{ \pm}$on $X$ such that

$$
\omega_{ \pm}(A)=\int_{X} d v_{ \pm}(\chi) \omega_{ \pm}^{\chi}(A), \text { for all } A \in \mathfrak{U},
$$

and for $v_{ \pm}$almost all $\chi \in X, \omega_{ \pm}^{\chi}$ is a pure state on $\mathfrak{A}$ satisfying spatial cluster properties, i.e.

$$
\lim _{|x| \rightarrow \infty} \omega_{ \pm}^{\chi}\left(A e^{i x P} B e^{-i x P}\right)=\omega_{ \pm}^{\chi}(A) \omega_{ \pm}^{\chi}(B) ;
$$

the theory reconstructed from $\omega_{ \pm}^{\chi}$ satisfies all Wightman axioms; $\omega_{ \pm}^{\chi}$ is locally Fock.

Proof. For the Wightman theory determined by $\omega_{ \pm}$the lemma has been proved in [18, Theorem 2.19] and [19, Theorem 1.4]. Since the Wightman theory coincides with the theory obtained from $\left(\omega_{ \pm}, \mathfrak{A}\right)$ by G.N.S. construction $[20,26]$, Lemma 6 follows from [19, Theorem 1.4, (3)]. See also [2]. The arguments proving the stability of the locally Fock property under the decomposition of Lemma 6 are standard. See [18, Section 2] for related results.

Conjecture. The states $\omega_{+}$and $\omega_{-}$are pure. They are the only pure vacuum states of the $(\boldsymbol{\phi} \cdot \boldsymbol{\phi})_{2}^{2}$-theory at $\mu=0$. 
It follows from the techniques of [28] that for large $\sigma, \omega_{+} \neq \omega_{-}$, and the $\phi_{1} \rightarrow-\phi_{1}$ symmetry is broken in the pure phases reconstructed from $\omega_{+}, \omega_{-}$.

We now let $\omega$ be an arbitrary pure vacuum state satisfying the cluster property (49) obtained from Lemma 6 . For such a state we may let $n$ tend to $\infty$ and complete the proof of Theorem 3 .

\section{Lemma 7.}

(1) $\lim _{n \rightarrow \infty} \omega\left(V_{\xi}^{*}\left(\alpha^{e, n}\right) \varrho_{\alpha^{e, n}}(A) V_{\xi}\left(\alpha^{e, n}\right)\right)$

$=\omega\left(V_{\xi}^{*}\left(\alpha^{e}\right) \varrho_{\alpha^{e}}(A) V_{\xi}\left(\alpha^{e}\right)\right)$

$\left.=\omega\left(\varrho_{\alpha} e^{(\tau-\xi}(A)\right)\right)$.

(2) $\lim _{n \rightarrow \infty} \omega\left(A V_{\xi}\left(\alpha^{e, n}\right) T_{\xi} B\right)$

$=\omega\left(A V_{\xi}\left(\alpha^{e}\right) T_{\xi} B\right) \omega\left(V_{\xi}\left(-\alpha^{e}\right)\right)$.

(3) $\left\{V_{\xi}\left(\alpha^{e}\right) T_{\xi} \mid \xi \in \mathbb{R}^{2}\right\}$ is a continuous unitary group on the vacuum sector $\mathscr{H}$ reconstructed from $(\omega, \mathfrak{U})$, the infinitesimal generators of which satisfy the relativistic spectrum condition. Proof. (1) It suffices to prove (1) for $A \in \bigcup_{\Lambda \in\{\Lambda\}} \mathfrak{U}(\Lambda)$. Then there exists a bounded
interval $\Lambda_{A}$ such that $A \in \mathfrak{A}\left(\Lambda_{A}\right)$.

By Eq. (44), Fact 5 and Lemma 3, (2)

$$
V_{\xi}\left(\alpha^{e, n}\right)=V_{t}\left(\alpha^{e, n}\right) \tau_{t}\left(e^{i L\left(\alpha^{e, n}-\alpha_{x}^{e, n}\right)}\right) \text {. }
$$

We let $|n|$ be so large that

and $\left.\begin{array}{l}\bar{\Lambda}_{\alpha^{e}, t} \cap \bar{\Lambda}_{\alpha_{n}^{e}, t}=\emptyset, \\ \Lambda_{\alpha^{e}-\alpha_{x}^{e}, t} \cap \bar{\Lambda}_{\alpha_{n}^{e}, t}=\emptyset .\end{array}\right\}$

Then by Lemma 3, (4)

$$
\begin{aligned}
& V_{t}\left(\alpha^{e, n}\right) \tau_{t}\left(e^{i L\left(\alpha^{e, n}-\alpha_{x}^{e}, n\right)}\right) \\
& =V_{t}\left(\alpha^{e}\right) V_{t}\left(-\alpha_{n}^{e}\right) \tau_{t}\left(e^{i L\left(\alpha^{e}-\alpha_{x}^{e}\right)}\right) \tau_{t}\left(e^{i L\left(-\alpha_{n}^{e}+\alpha_{n}^{e}+x\right)}\right) \\
& =V_{t}\left(\alpha^{e}\right) \tau_{t}\left(e^{i L\left(\alpha^{e}-\alpha_{x}^{e}\right)}\right) V_{t}\left(-\alpha_{n}^{e}\right) \tau_{t}\left(e^{i L\left(-\alpha_{n}^{e}+\alpha_{n}^{e}+x\right)}\right) \\
& =V_{\xi}\left(\alpha^{e}\right) V_{\xi}\left(-\alpha_{n}^{e}\right) .
\end{aligned}
$$

Let $d_{A}$ be the smallest real number such that $\Lambda_{A} \subseteq\left[-d_{A}, d_{A}\right]$ and $d_{\alpha^{e}}$ the smallest real number such that

$$
\operatorname{supp}\left(\partial_{x} \alpha^{e}\right)_{, t} \subseteq\left[-\frac{d_{\alpha^{e}}}{2}+x, \frac{d_{\alpha^{e}}}{2}-x\right] .
$$

Now pick $n$ such that (50) holds and

$$
n \geqq d_{A}+d_{\alpha^{e}} \text {. }
$$

Then $A$, thus $\varrho_{\alpha^{e}, n}(A)$, commute with $V_{\xi}\left(\alpha_{n}^{e}\right)$; moreover $\varrho_{\alpha^{e}, n}(A)=\varrho_{\alpha^{e}}(A)$. Hence

$$
\begin{aligned}
& V_{\xi}^{*}\left(\alpha^{e, n}\right) \varrho_{\alpha^{e, n}}(A) V_{\xi}\left(\alpha^{e, n}\right) \\
& =V_{\xi}^{*}\left(\alpha^{e}\right) V_{\xi}^{*}\left(-\alpha_{n}^{e}\right) \varrho_{\alpha^{e}}(A) V_{\xi}\left(-\alpha_{n}^{e}\right) V_{\xi}\left(\alpha^{e}\right) \\
& =V_{\xi}^{*}\left(\alpha^{e}\right) \varrho_{\alpha^{e}}(A) V_{\xi}\left(\alpha^{e}\right) .
\end{aligned}
$$


Due to the locally Fock property of $\omega$ and Lemma 3, (3), the operator on the r.h.s. of (53) can be further studied on the Fock space $\mathscr{F}$ using the spatially cutoff $(\phi \cdot \phi)_{2}^{2}$-Hamiltonian $H_{l}$. If we choose the cutoff $l$ large enough

$$
V_{\xi}^{*}\left(\alpha^{e}\right) \varrho_{\alpha^{e}}(A) V_{\xi}\left(\alpha^{e}\right)=V_{\xi}^{*}\left(\alpha^{e} \mid l\right) \varrho_{\alpha^{e}}(A) V_{\xi}\left(\alpha^{e} \mid l\right),
$$

by Eq. (44), Lemma 3, (3), Lemma 2, (1), and Fact 5. Using Definition 3 and Eq. (44) it is then trivial to show that

$$
V_{\xi}^{*}\left(\alpha^{e} \mid l\right) \varrho_{\alpha^{e}}(A) V_{\xi}\left(\alpha^{e} \mid l\right)=\tau_{\xi}\left(\varrho_{\alpha^{e}}\left(\tau_{-\xi}(A)\right)\right) .
$$

Since $\omega$ is invariant under $\tau_{\xi}$ this yields (1).

(2) By (50) and (51)

$$
V_{\xi}\left(\alpha^{e, n}\right)=V_{\xi}\left(\alpha^{e}\right) V_{\xi}\left(-\alpha_{n}^{e}\right) \text {. }
$$

Thus

$$
\begin{aligned}
\lim _{n \rightarrow \infty} \omega\left(A V_{\xi}\left(\alpha^{e, n}\right) T_{\xi} B\right) & =\lim _{n \rightarrow \infty} \omega\left(A V_{\xi}\left(\alpha^{e}\right) V_{\xi}\left(-\alpha_{n}^{e}\right) \tau_{\xi}(B)\right) \\
& \left.=\omega(A) V_{\xi}\left(\alpha^{e}\right) \tau_{\xi}(B)\right) \omega\left(V_{\xi}\left(-\alpha^{e}\right)\right),
\end{aligned}
$$

as a consequence of the cluster properties (49).

This proves (2).

(3) We first verify the group property for $\left\{V_{\xi}\left(\alpha^{e}\right) T_{\xi} \mid \xi \in \mathbb{R}^{2}\right\}$ :

$$
\begin{aligned}
& \omega\left(A V_{\xi_{1}}\left(\alpha^{e}\right) T_{\xi_{1}} V_{\xi_{2}}\left(\alpha^{e}\right) T_{\xi_{2}} B\right) \\
& =\omega\left(A V_{\xi_{1}}\left(\alpha^{e}\right) \tau_{\xi_{1}}\left(V_{\xi_{2}}\left(\alpha^{e}\right)\right) \tau_{\xi_{1}+\xi_{2}}(B)\right) .
\end{aligned}
$$

For the proof of the group property it suffices to choose $A$ and $B$ in $\mathfrak{A}(\Lambda)$ for some bounded, but otherwise arbitrary interval $\Lambda$, since $V_{\xi}\left(\alpha^{e}\right)$ and $T_{\xi}$ are unitary operators on $\mathscr{H}$.

We may then apply Lemma 3, (3) the locally Fock property of the state $\omega$ and Facts 4 and 5 to conclude that we may further analyze

$$
A V_{\xi_{1}}\left(\alpha^{e}\right) \tau_{\xi_{1}}\left(V_{\xi_{2}}\left(\alpha^{e}\right)\right) \tau_{\xi_{1}+\xi_{2}}(B)
$$

as an operator on the Fock space $\mathscr{F}$ using the spatially cutoff Hamiltonian $H_{l}$ and choosing $l$ so large that

$$
\begin{aligned}
& A V_{\xi_{1}}\left(\alpha^{e} \mid l\right) \tau_{\xi_{1}}\left(V_{\xi_{2}}\left(\alpha^{e} \mid l\right)\right) \tau_{\xi_{1}+\xi_{2}}(B) \\
& =A V_{\xi_{1}}\left(\alpha^{e}\right) \tau_{\xi_{1}}\left(V_{\xi_{2}}\left(\alpha^{e}\right)\right) \tau_{\xi_{1}+\xi_{2}}(B) \text { is independent of } l .
\end{aligned}
$$

We then get immediately

$$
\begin{aligned}
& A V_{\xi_{1}}\left(\alpha^{e}\right) \tau_{\xi_{1}}\left(V_{\xi_{2}}\left(\alpha^{e}\right)\right) \tau_{\xi_{1}+\xi_{2}}(B) \\
& =A V_{\xi_{1}+\xi_{2}}\left(\alpha^{e}\right) \tau_{\xi_{1}+\xi_{2}}(B) .
\end{aligned}
$$

Thus

$$
\begin{aligned}
& \omega\left(A V_{\xi_{1}}\left(\alpha^{e}\right) T_{\xi_{1}} V_{\xi_{2}}\left(\alpha^{e}\right) T_{\xi_{2}} B\right) \\
& =\omega\left(A V_{\xi_{1}+\xi_{2}}\left(\alpha^{e}\right) \tau_{\xi_{1}+\xi_{2}}(B)\right) \\
& =\omega\left(A V_{\xi_{1}+\xi_{2}}\left(\alpha^{e}\right) T_{\xi_{1}+\xi_{2}} B\right),
\end{aligned}
$$

(by the invariance of $\omega$ under $\tau_{\xi}$ ). 
This proves the group property.

Next we prove continuity of $V_{\xi}\left(\alpha^{e}\right) T_{\xi}$ in $\xi$ on $\mathscr{H}$. Continuity of $T_{\xi}$ in $\xi$ on $\mathscr{H}$ is known. By Eq. (44) and the locally Fock property

$$
V_{\xi}\left(\alpha^{e}\right)=V_{t}\left(\alpha^{e}\right) \tau_{t}\left(e^{i L\left(\alpha^{e}-\alpha_{x}^{e}\right)}\right) .
$$

Strong continuity of $V_{t}\left(\alpha^{e}\right)$ in $t$ on $\mathscr{F}$ has been proved in Lemma 3, (3). For $l$ large enough

$$
\tau_{t}\left(e^{i L\left(\alpha^{e}-\alpha_{x}^{e}\right)}\right)=e^{i t H_{l}}\left(e^{i L\left(\alpha^{e}-\alpha_{x}^{e}\right)}\right) e^{-i t H_{l}}
$$

which is obviously strongly continuous in $t$ and $x$ on $\mathscr{F}$. Thus $V_{\xi}\left(\alpha^{e}\right)$ is strongly continuous in $\xi$ on $\mathscr{F}$, and, since $V_{\xi}\left(\alpha^{e}\right) \in \mathfrak{A}(\Lambda)$ for some bounded $\Lambda$, it is thus strongly continuous in $\xi$ on $\mathscr{H}$, by the locally Fock property.

Finally we must show that the infinitesimal generator of $\left\{V_{t}\left(\alpha^{e}\right) e^{i t H} \mid t \in \mathbb{R}\right\}$ is positive.

We let $A$ and $B$ be operators in $\mathfrak{A}(\Lambda)$, for some bounded, but otherwise arbitrary interval $\Lambda$ and set

$$
B_{n} \equiv e^{-i n P} B e^{i n P} \text {. }
$$

Consider the expectation value

$$
\begin{aligned}
F_{n}^{A, B}(t) & \equiv \omega\left(A B_{n} V_{t}\left(\alpha^{e, n}\right) e^{i t H} B_{n}^{*} A^{*}\right) \\
& =\omega\left(A B_{n} V_{t}\left(\alpha^{e, n}\right) \tau_{t}\left(B_{n}^{*} A^{*}\right)\right) .
\end{aligned}
$$

The set $\Lambda_{\alpha^{e, n}}=\Lambda_{\alpha^{e}-\alpha_{n}^{e}}$ is bounded for all $|n|<\infty$. Hence by Lemma 3, (2) and our assumptions on $A$ and $B$

$$
C_{n} \equiv A B_{n} V_{t}\left(\alpha^{e, n}\right) \tau_{t}\left(B_{n}^{*} A^{*}\right) \in \mathfrak{U}\left(\Lambda_{n}\right),
$$

for some bounded interval $\Lambda_{n}$.

By the locally Fock property of $\omega$ we may further analyze $C_{n}$ on the Fock space $\mathscr{F}$ using the spatially cutoff Hamiltonian $H_{l}$ with $l$ sufficiently large. Using Definition 3 and Lemma 3, (2) we obtain

$$
\begin{aligned}
C_{n} & =A B_{n} e^{i L\left(\alpha^{e, n}\right)} \tau_{t}\left(e^{-i L\left(\alpha^{e, n}\right)}\right) \tau_{t}\left(B_{n}^{*} A^{*}\right) \\
& =A B_{n} e^{i L\left(\alpha^{e, n}\right)} \tau_{t}\left(e^{-i L\left(\alpha^{e, n}\right)} B_{n}^{*} A^{*}\right) .
\end{aligned}
$$

Since $e^{i L\left(\alpha^{e, n}\right)} \in \mathfrak{U}\left(\Lambda_{\alpha^{e}, n}\right)$, we conclude that

$$
\begin{aligned}
F_{n}^{A, B}(t) & \equiv \omega\left(A B_{n} V_{t}\left(\alpha^{e, n}\right) e^{i t H} B_{n}^{*} A^{*}\right) \\
& =\omega\left(C_{n}\right)=\omega\left(A B_{n} e^{i L\left(\alpha^{e, n}\right)} \tau_{t}\left(e^{-i L\left(\alpha^{e, n}\right)} B_{n}^{*} A^{*}\right)\right) .
\end{aligned}
$$

Since $e^{i L\left(\alpha^{e, n}\right)}$ is unitary on $\mathscr{H}$ for all $|n|<\infty$ and $H$ is a positive operator, the Fourier transform of $F_{n}^{A, B}(t)$ is supported on the positive half line. We now let $n$ tend to $\infty$ :

Using the cluster property (49) and Lemma 3, (4) we get

$$
\begin{aligned}
\lim _{n \rightarrow \infty} F_{n}^{A, B}(t) & =\lim _{n \rightarrow \infty} \omega\left(A B_{n} V_{t}\left(\alpha^{e}\right) V_{t}\left(-\alpha_{n}^{e}\right) \tau_{t}\left(B_{n}^{*}\right) \tau_{t}\left(A^{*}\right)\right) \\
& =\omega\left(A V_{t}\left(\alpha^{e}\right) \tau_{t}\left(A^{*}\right)\right) \omega\left(B V_{t}\left(-\alpha^{e}\right) \tau_{t}\left(B^{*}\right)\right) \\
& =\omega\left(A V_{t}\left(\alpha^{e}\right) e^{i t H} A^{*}\right) \omega\left(B V_{t}\left(-\alpha^{e}\right) e^{i t H} B^{*}\right) .
\end{aligned}
$$


Since the Fourier transform of $\lim _{n \rightarrow \infty} F_{n}^{A, B}(t)$ is supported on the positive half line for arbitrary $A$ and $B$ in $\bigcup_{\Lambda \in\{\Lambda\}} \mathfrak{U}(\Lambda)$ the Fourier transform of $\omega\left(A V_{t}\left(\alpha^{e}\right) e^{i t H} A^{*}\right)$ and of $\omega\left(B V_{t}\left(-\alpha^{e}\right) e^{i t H} B^{*}\right)$ are supported on the positive half line, as well, for arbitrary $A$ and $B$. The relativistic spectrum condition for the generators of $\left\{V_{\xi}\left(\alpha^{e}\right) T_{\xi} \mid \xi \in \mathbb{R}^{2}\right\}$ follows by similar arguments. This completes the proof of (3).

Q.E.D.

Proof of Theorem 3. Let $\omega$ be an arbitrary pure phase state at $\mu=0$ or a direct integral of such states.

$\left.1^{\circ}\right)$ From $\left(\omega^{\circ} \varrho_{\alpha^{e}}, \mathfrak{A}\right)$ with $e= \pm 1$, we obtain a Hilbert space $\mathscr{H}_{\pi}^{e}$ and a representation $\pi_{\alpha^{e}}$ of $\mathfrak{A}$ on $\mathscr{H}_{\pi}^{e}$ by the G.N.S. construction.

$2^{\circ}$ ) By Lemma 7, (1) the state $\omega^{\circ} \varrho_{\alpha^{e}}$ is space-time translation covariant; for

$$
\begin{aligned}
\omega^{\circ} \varrho_{\alpha^{e}}\left(\tau_{\xi}(A)\right) & =\omega\left(\varrho_{\alpha^{e}}\left(\tau_{\xi}(A)\right)\right) \\
& =\omega\left(V_{-\xi}^{*}\left(\alpha^{e}\right) \varrho_{\alpha^{e}}(A) V_{-\xi}\left(\alpha^{e}\right)\right) .
\end{aligned}
$$

By Lemma $7,(3),\left\{V_{\xi}\left(\alpha^{e}\right) T_{\xi} \mid \xi \in \mathbb{R}^{2}\right\}$ is a strongly continuous unitary group on $\mathscr{H}$ so that $\omega^{\circ} \varrho_{\alpha} \circ \tau_{\xi}$ is a vector state of $B\left(\mathscr{H}_{\pi}^{e}\right)$ - the algebra of all bounded operators on $\mathscr{H}_{\pi}^{e}$ - and is continuous in $\xi$ in norm. Therefore there exists a unitary group $\left\{e^{i\left(t H_{\pi}^{e}-x P_{\pi}^{e}\right)} \mid(t, x) \in \mathbb{R}^{2}\right\}$ which implements the space-time translation automorphisms of $\mathfrak{A}$ on $\mathscr{H}_{\pi}^{e}$, and

$$
e^{i\left(t H_{\pi}^{e}-x P_{\pi}^{e}\right)} T_{\alpha} \Psi=T_{\alpha} V_{\xi}\left(\alpha^{e}\right) T_{\xi} \Psi,
$$

for all $\Psi \in \mathscr{H}$, which [with Eq. (44)] proves (22) and (23). Since $\left\{V_{\xi}\left(\alpha^{e}\right) T_{\xi} \mid \xi \in \mathbb{R}^{2}\right\}$ is an abelian group, we obtain (24).

$3^{\circ}$ ) By Lemma 7, (3)

$$
\begin{aligned}
F^{A}(t) & \equiv \omega^{\circ} \varrho_{\alpha^{e}}\left(A^{*} e^{i t H_{\pi}^{e}} A\right)=\omega\left(\varrho_{\alpha^{e}}\left(A^{*} e^{i t H_{\tau}^{e}} A\right)\right) \\
& =\omega\left(\varrho_{\alpha^{e}}\left(A^{*}\right) T_{\alpha^{e}}^{*} e^{i t H_{\pi}^{e}} T_{\alpha^{e}} \varrho_{\alpha^{e}}(A)\right) \\
& =\omega\left(\varrho_{\alpha^{e}}(A)^{*} V_{t}\left(\alpha^{e}\right) e^{i t H} \varrho_{\alpha^{e}}(A)\right)
\end{aligned}
$$

has a Fourier transform which is supported on the positive half line, for all $A \in \mathfrak{A}$, so that $H_{\pi}^{e} \geqq 0$. In the same way we obtain

$$
\operatorname{spec}\left(H_{\pi}^{e}, P_{\pi}^{e}\right) \subseteq \bar{V}_{+} .
$$

Remark. The procedure for the proof of Theorem 3 outlined in Lemmata 3, 6 , and 7 can be applied in a much more general, largely model-independent context, as will be indicated later.

Next we study the structure of the super-selection sectors constructed in Theorem 3. The main question is whether these sectors are really disjoint from the vacuum sector.

Let $\omega_{+}$be a pure vacuum state of the $(\phi \cdot \phi)_{2}^{2}$-theory satisfying the cluster property (49) such as obtained in Lemma 6.

Let $\left\{\alpha_{i}^{+}\right\}_{i=1}^{m+n}$ be kink functions satisfying the soliton-condition (46), (21). We set

$$
\underline{\alpha}^{+}(m) \equiv \sum_{j=1}^{m} \alpha_{j}^{+} \quad \text { and } \quad \underline{\alpha}^{-}(n) \equiv \sum_{j=m+1}^{m+n} \alpha_{j}^{-} .
$$


The group law of $S O(2)$ and definition (17) give

$$
\varrho_{\alpha_{1}^{+} \circ}^{\circ} \ldots \circ \varrho_{\alpha_{m}^{+} \circ} \varrho_{\alpha_{m}^{-}+1}{ }^{\circ} \ldots \varrho_{\alpha_{m+n}^{-}}=\varrho_{\alpha^{+}(m)+\underline{\alpha}^{-}(n)} .
$$

Let $\mathscr{H}_{+}$be the vacuum sector reconstructed from $\left(\omega_{+}, \mathfrak{U}\right)$.

Lemma 8. If $m$ and $n$ are even numbers, the automorphism

$$
\varrho_{\underline{\alpha}^{+}(m)+\underline{\alpha}^{-}(n)}
$$

is unitarily implementable on $\mathscr{H}_{+}$.

Proof. Since $m$ and $n$ are even numbers and the functions $\left\{\alpha_{i}^{+}\right\}_{i=1}^{m+n}$ satisfy the soliton-condition,

$$
\Lambda_{\underline{\alpha}^{+}(m)+\underline{\alpha}^{-}(m)}=\left\{x \mid\left[\underline{\alpha}^{+}(m)+\underline{\alpha}^{-}(n)\right](x) \neq 2 m \pi, m \in \mathbb{Z}\right\}^{-}
$$

is a compact set.

Therefore, by Lemma 2, (1)

$$
e^{i L\left(\underline{\alpha}^{+}(m)+\underline{\alpha}^{-}(m)\right)} \in \mathfrak{U}\left(\Lambda_{\underline{\alpha}^{+}(m)+\underline{\alpha}^{-}(n)}\right) .
$$

This operator implements $\varrho_{\alpha^{+}(m)+\alpha^{-}(n)}$ unitarily on $\mathscr{F}$. By the locally Fock property of the state $\omega_{+}$(see Lemma $\left.\frac{6}{6}\right), e^{i L\left(\alpha^{+}(m)+\alpha^{-}(n)\right)}$ determines a unique, unitary operator $T_{\underline{\alpha}^{+}(m)+\underline{\alpha}^{-}(n)} \in \mathfrak{U}\left(\Lambda_{\underline{\alpha}^{+}(m)+\underline{\alpha}^{-}(n)}\right)$ implementing $\varrho_{\underline{\alpha}^{+}(m)+\underline{\alpha}^{-}(n)}$ unitarily on $\mathscr{H}_{+}$. Q.E.D.

Due to the composition law (54) and Lemma 8, it now suffices to study the automorphisms $\varrho_{\underline{\alpha}^{+}}$and $\varrho_{\underline{\alpha}^{-}}$with $\alpha^{+}$satisfying the soliton-condition.

A priori it is not clear whether the automorphisms $\varrho_{\alpha^{ \pm}}$are unitarily implemented on $\mathscr{H}_{+}$or not - they are implemented on the Fock space $\mathscr{F}$. If the $\phi_{1} \rightarrow-\phi_{1}$ symmetry of the $(\phi \cdot \phi)_{2}^{2}$-Lagrangian is spontaneously broken by the vacuum state $\omega_{+}$we can prove that $\varrho_{\alpha^{+}}$and $\varrho_{\alpha^{-}}$are not unitarily implemented on $\mathscr{H}_{+}$. Thanks to the new results of [28] one may show that if the coefficient $\sigma$ of the term : $\phi_{1}^{2}$ : in the Lagrangian is large enough the $\phi_{1} \rightarrow-\phi_{1}$ symmetry is spontaneously broken by $\omega_{+}$and that $\omega_{+}\left(\phi_{1}\right)>0$. This is assumed henceforth. By Lemma 6 we may suppose that $\omega_{+}$is a pure phase state satisfying the cluster property (49) and we may also assume that

$$
\omega_{+}\left(\phi_{1}(x, t)\right)=\left\langle\Omega_{+}, \phi_{1}(x, t) \Omega_{+}\right\rangle \equiv \phi_{c}>0 .
$$

The state $\omega_{-} \equiv \omega_{+}{ }^{\circ} \varrho_{\pi}$ is then a translation-invariant pure phase state satisfying (49), and

$$
\omega_{-}\left(\phi_{1}(x, t)\right)=-\phi_{c}<0 .
$$

If $\alpha_{1}^{+}$and $\alpha_{2}^{+}$satisfy the soliton-condition then $\varrho_{\pi^{\circ}}^{\circ} \varrho_{\alpha_{1}^{+}} \varrho_{\alpha_{2}^{-}}$is unitarily implemented on $\mathscr{H}_{+}$, by Lemma 8 .

Therefore there exists a unitary operator $T_{\alpha_{1}^{+}+\alpha_{2}^{-}}$on the vacuum sector $\mathscr{H}_{-}$ reconstructed from $\left(\omega_{-}, \mathfrak{A}\right)$ such that

$$
\left\langle T_{\alpha_{1}^{+}+\alpha_{2}^{-}} \Omega_{-}, \circ T_{\alpha_{1}^{+}+\alpha_{2}^{-}} \Omega_{-}\right\rangle=\omega_{+}{ }^{\circ} \varrho_{\alpha_{1}^{+}+\alpha_{2}^{-}}(\cdot) .
$$

We define the Hilbert spaces

$$
\mathscr{H}_{\pi}^{+}=\left\{T_{\alpha^{+}} \Psi \mid \Psi \in \mathscr{H}_{+}, \alpha_{\infty}^{+}=\pi\right\},
$$


and

$$
\begin{aligned}
\mathscr{H}_{\pi}^{-} & =\left\{T_{\alpha^{-}} \Psi \mid \Psi \in \mathscr{H}_{+}, \alpha_{\infty}^{+}=\pi\right\} \\
& =\left\{T_{\alpha^{+}} \Psi \mid \Psi \in \mathscr{H}_{-}, \alpha_{\infty}^{+}=\pi\right\} .
\end{aligned}
$$

Theorem 4. Assume that $\sigma \gg 1$ and that the vacuum state $\omega_{+}$is chosen such as described above.

Then the $(\phi \cdot \phi)_{2}^{2}$-theory has four orthogonal super-selection sectors $\mathscr{H}_{+}, \mathscr{H}_{-}$, $\mathscr{H}_{\pi}^{+}$, and $\mathscr{H}_{\pi}^{-}$. The representations of the algebra $\mathfrak{A}$ on $\mathscr{H}_{+}, \mathscr{H}_{-}, \mathscr{H}_{\pi}^{+}, \mathscr{H}_{\pi}^{-}$are pairwise unitarily inequivalent (more precisely disjoint). in $\mathscr{H}_{+}$

The spectrum of $P_{\pi}^{ \pm}$is purely continuous. For all vectors $\Psi$ in a subspace dense

$$
\left\langle T_{\alpha^{ \pm}} \Psi, \phi_{1}(x, t) T_{\alpha^{ \pm}} \Psi\right\rangle \rightarrow\left\{\begin{array}{l} 
\pm\langle\Psi, \Psi\rangle \phi_{c}, \text { as } x \rightarrow-\infty \\
\mp\langle\Psi, \Psi\rangle \phi_{c}, \text { as } x \rightarrow+\infty
\end{array}\right.
$$

i.e. parity is spontaneously broken on $\mathscr{H}_{\pi}^{ \pm 3}$.

Remarks. Obviously the behaviour qualitatively described in (60) reminds one very much of the behaviour of the soliton-solutions of the classical, non-linear field equation, even though $\left\langle T_{\alpha^{ \pm}} \Psi, \phi_{1}(x, t) T_{\alpha^{ \pm}} \Psi\right\rangle$ is never strictly stationary in $t$. That is why we call $\mathscr{H}_{\pi}^{+}$the "soliton-" and $\mathscr{H}_{\pi}^{-}$the "anti-soliton" sector.

As a consequence of Lemma 8 and (58) we have: For $\Psi \in \mathscr{H}_{+}$and $m, n$ arbitrary

$$
\left.\begin{array}{l}
T_{\underline{\alpha}^{+}(2 m)+\underline{\alpha}^{-}(2 n)} \Psi \in \mathscr{H}_{+}, T_{\underline{\alpha}^{+}(2 m+1)+\underline{\alpha}^{-}(2 n)} \Psi \in \mathscr{H}_{\pi}^{+}, \\
T_{\underline{\alpha}^{+}(2 m)+\underline{\alpha}^{-}(2 n+1)} \Psi \in \mathscr{H}_{\pi}^{-}, T_{\underline{\alpha}^{+}(2 m+1)+\underline{\alpha}^{-}(2 n+1)} \Psi \in \mathscr{H}_{-}
\end{array}\right\}
$$

Proof of Theorem 4. The fact that $\mathscr{H}_{+}$and $\mathscr{H}_{-}$are orthogonal and that the representations of $\mathfrak{U}$ on $\mathscr{H}_{+}$and $\mathscr{H}_{-}$are disjoint is well known [2].

Let $f$ be some positive test function on $\mathbb{R}^{2}$ of compact support and let $f_{x, t}(y, s) \equiv$ $f(y-x, s-t)$. Then for all $\Psi \in \mathscr{H}_{ \pm}$

$$
\begin{aligned}
\lim _{x \rightarrow \infty}\left\langle\Psi, e^{i \phi_{1}\left(f_{x, t}\right)} \Psi\right\rangle & =\lim _{x \rightarrow-\infty}\left\langle\Psi, e^{i \phi_{1}\left(f_{x, t}\right)} \Psi\right\rangle \\
& =\langle\Psi, \Psi\rangle\left\langle\Omega_{ \pm}, e^{i \phi_{1}(f)} \Omega_{ \pm}\right\rangle,
\end{aligned}
$$

which is an immediate consequence of the cluster property (49). However for all $\Psi \in \mathscr{H}_{+}$

$$
\begin{aligned}
& \lim _{x \rightarrow \infty}\left\langle T_{\alpha^{ \pm}} \Psi, e^{i \phi_{1}\left(f_{x, t}\right)} T_{\alpha^{ \pm}} \Psi\right\rangle \\
& =\lim _{x \rightarrow-\infty}\left\langle T_{\alpha^{ \pm}} \Psi, e^{-i \phi_{1}\left(f_{x, t}\right)} T_{\alpha^{ \pm}} \Psi\right\rangle \\
& =\langle\Psi, \Psi\rangle\left\langle\Omega_{+}, e^{\mp i \phi_{1}(f)} \Omega_{+}\right\rangle,
\end{aligned}
$$

by definition (17) of $\varrho_{\alpha^{ \pm}}$and $T_{\alpha^{ \pm}}$, the unitarity of $\left\{e^{i t H_{\pi}^{ \pm}} \mid t \in \mathbb{R}\right\}$ on $\mathscr{H}_{\pi}^{ \pm}-$see Theorem 3, (22) - and by (62). Obviously the r.h.s. of (63) is independent of $t$.

The factor $\langle\Psi, \Psi\rangle$ on the r.h.s. of (63) is real, but the second factor on the r.h.s. of (63) is not, since $\omega_{+}$breaks the $\phi_{1} \rightarrow-\phi_{1}$ symmetry [see (55), (56)]. Thus, for $\Psi \neq \overrightarrow{0}$,

$$
\lim _{x \rightarrow \infty}\left\langle T_{\alpha^{ \pm}} \Psi, e^{i \phi_{1}\left(f_{x, t}\right)} T_{\alpha^{ \pm}} \Psi\right\rangle \neq \lim _{x \rightarrow-\infty}\langle\cdots\rangle .
$$

Lorentz covariance of $\mathscr{H}_{\pi}^{ \pm}$has recently been proven; see Remark $7, \S 6$. 
From (49) and (55) we conclude that for all $\Psi$ in some subspace dense in $\mathscr{H}_{+}$

$$
\begin{aligned}
& \left\langle T_{\alpha^{ \pm}} \Psi, \phi_{1}(x, t) T_{\alpha^{ \pm}} \Psi\right\rangle \\
& =\left\langle\Psi, \varrho_{\alpha^{ \pm}}\left(\phi_{1}(x, t)\right) \Psi\right\rangle \rightarrow\left\{\begin{array}{l}
\mp\langle\Psi, \Psi\rangle \phi_{c}, x \rightarrow+\infty \\
\pm\langle\Psi, \Psi\rangle \phi_{c}, x \rightarrow-\infty .
\end{array}\right.
\end{aligned}
$$

Formulas (62) and (64) obviously prove that $\mathscr{H}_{+}$and $\mathscr{H}_{\pi}^{ \pm}$, and $\mathscr{H}^{-}$and $\mathscr{H}_{\pi}^{ \pm}$are orthogonal. The orthogonality of $\mathscr{H}_{\pi}^{+}$and $\mathscr{H}_{\pi}^{-}$follows from (63) (and, alternatively, from the one of $\mathscr{H}_{+}$and $\mathscr{H}_{-}$). In more precise terms, formulas (62)-(64) prove that the representations of $\mathscr{A}$ on $\mathscr{H}_{+}, \mathscr{H}_{\pi}^{+}$and $\mathscr{H}_{\pi}^{-}$are pairwise inequivalent. It is known that the representations of $\mathfrak{A}_{\text {on }} \mathscr{H}_{+}$and $\mathscr{H}_{-}$are irreducible, see [2]. Since $\varrho_{\alpha^{ \pm}}$are invertible ${ }^{*}$-automorphisms of $\mathfrak{A}$, the representations of $\mathfrak{A}$ on $\mathscr{H}_{\pi}^{+}$ and $\mathscr{H}_{\pi}^{-}$are irreducible, as well. Hence the representations of $\mathfrak{U}$ on $\mathscr{H}_{+}, \mathscr{H}_{\pi}^{+}, \mathscr{H}_{-}$, and $\mathscr{H}_{\pi}^{-}$are pairwise disjoint. The breaking of parity on $\mathscr{H}_{\pi}^{ \pm}$follows directly from (64).

Finally we learn from (63) and (64) that for all $\overrightarrow{0} \neq \Psi \in \mathscr{H}_{+}$and a positive test function $f$ of compact support

$$
\begin{aligned}
& \lim _{x \rightarrow \infty}\left\langle T_{\alpha^{ \pm}} \Psi, e^{-i x P_{\pi}^{ \pm}} e^{i \phi_{1}(f)} e^{+i x P_{\pi}^{ \pm}} T_{\alpha^{ \pm}} \Psi\right\rangle \\
& =\lim _{x \rightarrow \infty}\left\langle T_{\alpha^{ \pm}} \Psi, e^{i \phi_{1}\left(f_{x}, 0^{o}\right)} T_{\alpha^{ \pm}} \Psi\right\rangle \\
& \neq \lim _{x \rightarrow-\infty}\left\langle T_{\alpha^{ \pm}} \Psi, e^{i \phi_{1}\left(f_{x}, 0\right)} T_{\alpha^{ \pm}} \Psi\right\rangle \\
& =\lim _{x \rightarrow-\infty}\left\langle T_{\alpha^{ \pm}} \Psi, e^{i x P_{\pi}^{ \pm}} \ldots\right\rangle .
\end{aligned}
$$

Therefore $P_{\pi}^{ \pm}$does not have any discrete spectrum.

Q.E.D.

Remarks. If the $(\phi \cdot \phi)_{2}^{2}$-theory is Lorentz-covariant on the sectors $\mathscr{H}_{\pi}^{ \pm}$then it follows from the continuity of $\operatorname{spec} P_{\pi}^{ \pm}$that $\operatorname{spec} H_{\pi}^{ \pm}$is purely continuous, as well. Although the functions $\varphi_{\alpha^{ \pm}, \Psi}(x, t) \equiv\left\langle T_{\alpha^{ \pm}} \Psi, \phi_{1}(x, t) T_{\alpha^{ \pm}} \Psi\right\rangle$ [see Theorem 4, (60)], remind one of the soliton-solutions of the classical field equation there is one essential difference: If the $(\phi \cdot \phi)_{2}^{2}$-theory is Lorentz-covariant on $\mathscr{H}_{\pi}^{ \pm}$it follows from the continuity of $\operatorname{spec} H_{\pi}^{ \pm}$that the function $\varphi_{\alpha^{ \pm}, \Psi}(x, t)$ is never strictly stationary in $t$.

There may exist states $\varrho$ on $\mathfrak{U}$ with the property that $\varrho\left(\phi_{1}(x, t)\right)$ is similar to the function $\varphi_{\alpha^{ \pm}, \Psi}(x, t)$ but is independent of $t\left[\varrho\left(\phi_{1}(x, t)\right)\right.$ is the strict analogue of the time-independent, classical soliton solution]. The state $\varrho$ is then, however, a non-translation invariant vacuum state which has to be sharply distinguished from a soliton state: see Section 6.

\section{§ 4. The Scattering of Solitons}

Next we examine the consequences of assuming the existence of one-(anti-) soliton states with discrete mass for the physics on the vacuum sector.

Definition 6. We define as the physical Hilbert space of the $(\boldsymbol{\phi} \cdot \boldsymbol{\phi})_{2}^{2}$-theory

$$
\mathscr{H}_{\text {phys. }} \equiv \mathscr{H}_{+} \oplus \mathscr{H}_{-} \oplus \mathscr{H}_{\pi}^{+} \oplus \mathscr{H}_{\pi}^{-} .
$$


The total energy-momentum operator is given by

$$
(\mathbb{H}, \mathbb{P}) \equiv(H, P)\left|\left(\mathscr{H}_{+} \oplus \mathscr{H}_{-}\right) \oplus\left(H_{\pi}^{+}, P_{\pi}^{+}\right)\right| \mathscr{H}_{\pi}^{+} \oplus\left(H_{\pi}^{-}, P_{\pi}^{-}\right) \mid \mathscr{H}_{\pi}^{-} .
$$

Let $\alpha^{+}$be a kink function satisfying the soliton-condition (see Definition 5) and let $\alpha^{-}(x)=\alpha^{+}(-x)$. We define soliton- and anti-soliton fields $s, \bar{s}$ by

$$
\begin{aligned}
& s(x, t) \equiv e^{i(t \mathbb{H}-x \mathbb{P})} T_{\alpha^{+}} e^{-i(t \mathbb{H}-x \mathbb{P})} \\
& \bar{s}(x, t) \equiv e^{i(t \mathbb{H}-x \mathbb{P})} T_{\alpha^{-}} e^{-i(t \mathbb{H}-x \mathbb{P})},
\end{aligned}
$$

and $s^{e}=\left\{\begin{array}{l}s, \text { for } e=+1 \\ \bar{s}, \text { for } e=-1\end{array}\right.$

\section{Clearly}

$$
s^{e}(\xi)=T_{\alpha^{e}} V_{\xi}\left(\alpha^{e}\right)=T_{\alpha_{x}^{e}} V_{t}\left(\alpha_{x}^{e}\right) \text {, with } \xi=(x, t) .
$$

From Eq. (68) we may derive locality properties: Suppose that

$$
\bar{\Lambda}_{\alpha_{x}, t} \cap \bar{\Lambda}_{\alpha_{x^{\prime}} e^{\prime}, t^{\prime}}=\emptyset \text {. }
$$

Then

$$
\begin{aligned}
s^{e_{1}}(\xi) s^{e_{2}}\left(\xi^{\prime}\right) & =T_{\alpha_{x}^{1}+\alpha_{x^{\prime}}^{e_{2}}}^{e_{\alpha_{x^{\prime}}}^{e_{2}}}\left(V_{t}\left(\alpha_{x}^{e_{1}}\right)\right) V_{t^{\prime}}\left(\alpha_{x^{\prime}}^{e_{2}}\right) \\
& =T_{\alpha_{x}+e_{x^{\prime}}^{e_{1}^{\prime}}}^{e_{2}} V_{t}\left(\alpha_{x}^{e_{1}}\right) V_{t^{\prime}}\left(\alpha_{x}^{e_{2}}\right),
\end{aligned}
$$

since by (69)

$$
\varrho_{\alpha_{x^{\prime}}}^{e_{2}}\left(V_{t}\left(\alpha_{x}^{e_{1}}\right)\right)=\left\{\begin{array}{l}
V_{t}\left(\alpha_{x}^{e_{1}}\right), \text { or } \\
\varrho_{\pi}\left(V_{t}\left(\alpha_{x}^{e_{1}}\right)\right)
\end{array}\right.
$$

and $\varrho_{\pi}\left(V_{t}\left(\alpha_{x}^{e_{1}}\right)\right)=V_{t}\left(\alpha_{x}^{e_{1}}\right)$, by the $\phi \mapsto-\phi$ symmetry of $(\mathbb{H}, \mathbb{P})$. Therefore

$$
\begin{aligned}
& s^{e_{1}}(\xi) s^{e_{2}}\left(\xi^{\prime}\right)=T_{\alpha_{x}^{e_{1}}+\alpha_{x^{\prime}}^{e_{2}}} V_{t}\left(\alpha_{x}^{e_{1}}\right) V_{t^{\prime}}\left(\alpha_{x^{\prime}}^{e_{2}}\right) \\
& =T_{\alpha_{x^{\prime}}}^{e_{2}} T_{\alpha_{x}}^{e_{1}} V_{t^{\prime}}\left(\alpha_{x^{\prime}}^{e_{2}}\right) V_{t}\left(\alpha_{x}^{e_{1}}\right) \text {, by (69) and Lemma 3, (3) } \\
& =T_{\alpha_{x^{\prime}}}^{e_{2}} Q_{-\alpha_{x}}\left(V_{t^{\prime}}\left(\alpha_{x^{\prime}}^{e_{2}}\right)\right) T_{\alpha_{x}^{e_{1}}} V_{t}\left(\alpha_{x}^{e_{1}}\right) \\
& =T_{\alpha_{x^{\prime}}}^{e_{2}} V_{t^{\prime}}\left(\alpha_{x^{\prime}}^{e_{2}}\right) T_{\alpha_{x}^{1}}^{e_{1}^{1}} V_{t}\left(\alpha_{x}^{e_{1}}\right) \\
& =s^{e_{2}}\left(\xi^{\prime}\right) s^{e_{1}}(\xi) \text {, by }(68) \text {. }
\end{aligned}
$$

Since $\operatorname{supp}\left(\partial_{x} \alpha^{+}\right)$may be an arbitrarily small interval, the soliton- and antisoliton fields may be chosen so that they are almost local relative to each other. They are, however, "anti-local" relative to the fundamental field $\phi$ :

In general

$$
\begin{aligned}
s^{e}(\xi) \phi_{i}\left(\xi^{\prime}\right)= & e^{i(t \mathbb{H}-x \mathbb{P})} T_{\alpha^{e}} \phi_{i}\left(\xi^{\prime}-\xi\right) e^{-i(t \mathbb{H}-x \mathbb{P})} \\
= & {\left[e^{i(t \mathbb{H}-x \mathbb{P})} T_{\alpha^{e}} \phi_{i}\left(\xi^{\prime}-\xi\right) T_{\alpha^{e}}^{*} e^{-i(t \mathbb{H}-x \mathbb{P})}\right] } \\
& \times\left[e^{i(t \mathbb{H}-x \mathbb{P})} T_{\alpha^{e}} e^{-i(t \mathbb{H}-x \mathbb{P})}\right] \\
= & \tau_{\xi}\left(\varrho_{-\alpha^{e}}\left(\phi_{i}\left(\xi^{\prime}-\xi\right)\right)\right) s^{e}(\xi) .
\end{aligned}
$$


Therefore if $\left\{x^{\prime}\right\}_{t^{\prime}} \cap \bar{\Lambda}_{\alpha_{x, t}^{e}}=\emptyset$

$$
s^{e}(\xi) \phi_{i}\left(\xi^{\prime}\right)=\left\{\begin{aligned}
&-\phi_{i}\left(\xi^{\prime}\right) s^{e}(\xi) \text { if } \alpha^{e}=\pi \text { on }\left\{x^{\prime}\right\}_{t^{\prime}} \\
& \phi_{i}\left(\xi^{\prime}\right) s^{e}(\xi) \text { if } \alpha^{e}=0 \text { on }\left\{x^{\prime}\right\}_{t^{\prime}} .
\end{aligned}\right.
$$

Clearly the soliton- and anti-soliton fields are almost local relative to even, local functions of $\phi$.

We now consider arbitrary vacuum expectation values (v.e.v.'s) of the fields $s, \bar{s}, \phi_{1}, \phi_{2}$. For the purposes of scattering theory we must define the truncated v.e.v.'s, too. The truncation is more complicated than in the case of a field theory with local fields and a unique vacuum. This is because according to (61) odd numbers of soliton- and of anti-soliton fields map $\mathscr{H}_{ \pm}$onto $\mathscr{H}_{\mp}$, so that when defining truncation we always have to take into account two possible intermediate vacua, $\Omega_{+}$and $\Omega_{-}$.

Let $\pi$ be an arbitrary product of $s, \bar{s}, \phi_{1}$, and $\phi_{2}$ fields, $n$ in number. We consider arbitrary proper partitions $p$ of $\pi$ into disjoint clusters $C_{1}^{p}, \ldots, C_{n}^{p}$, where some of these clusters may be empty, and such that each cluster contains an odd or an even number of $s$ and of $\bar{s}$ fields. We write

$$
\pi=\Psi_{j_{1}}^{p}(1) \ldots \Psi_{j_{n}}^{p}(n),
$$

where $\Psi(l)$ is an $s, \bar{s}, \phi_{1}$ or $\phi_{2}$ field, and $\Psi_{j_{l}}^{p}(l)$ belongs to cluster $C_{j_{l}}^{p}$.

We set

$$
\Psi_{\xi}(l)=e^{i(t \mathbb{H}-x \mathbb{P})} \Psi(l) e^{-i(t \mathbb{H}-x \mathbb{P})} .
$$

Suppose that $\pi$ has non-vanishing matrix elements between $\mathscr{H}_{+}$and $\mathscr{H}_{e(\pi)}$, where $e(\pi)=+$ if $\pi$ contains an even number of $s$ and $\bar{s}$ fields and $e(\pi)=-$ if $\pi$ contains an odd number of these fields. Assume that the physical mass

$$
m_{*} \equiv \operatorname{dist}(0, \operatorname{spec} \mathbb{H} \backslash\{0\})
$$

is positive. Let $\chi_{n}(\xi)$ be a Schwartz space function whose Fourier transform is supported on $\left\{\left(p, p^{0}\right) \mid p^{2}+\left(p^{0}\right)^{2} \leqq m_{*} / n+1\right\}$, and $\tilde{\chi}_{n}(0)=1$.

We now define

$$
\begin{aligned}
& \left\langle\Omega_{+}, \pi \Omega_{e(\pi)}\right\rangle^{T}=\left\langle\Omega_{+}, \pi \Omega_{e(\pi)}\right\rangle \\
& +\sum_{p}(-1)^{|p|-1}(|p|-1) ! \int \prod_{j=1}^{n} d^{2} \xi_{j} \chi_{n}\left(\xi_{j}\right)\left\langle\Omega_{+}, \Psi_{j_{1}, \xi_{j_{1}}}^{p}(1) \ldots \Psi_{j_{n}, \xi_{j_{n}}}^{p}(n) \Omega_{e(\pi)}\right\rangle,
\end{aligned}
$$

where $|p|$ is the number of non-empty clusters in $p$.

It is to be noticed that because of the commutation relations (71) the truncated v.e.v.'s as defined in (73) do in general not decay to 0 , as the separation between two of their arguments tends to $\infty$. This can be seen by considering e.g.

$$
\left\langle\Omega_{+}, s\left(\xi_{1}\right) \phi_{1}\left(\xi_{2}\right) s\left(\xi_{3}\right) \Omega_{+}\right\rangle^{T}
$$

and letting $\xi_{2}$ tend to $\pm \infty$.

If there exist one-soliton and one-anti-soliton states with discrete mass it is, however, consistent to assume that the Fourier transforms of the truncated v.e.v.'s have sufficient smoothness properties in some neighbourhood of the mass shells 
to permit us to construct the scattering theory on $\mathscr{H}_{\text {phys. }}$ à la Haag-Ruelle for non-overlapping test-functions [29] vanishing at the origin in momentum space. At this point no new concepts are required, and (on a formal level) one may proceed as in [29]. Since the analysis of spec $(\mathbb{H}, \mathbb{P})$ has not advanced enough, details are deferred to later investigations.

We now assume that such a scattering theory for solitons, anti-solitons and mesons exists. Let $N_{s}$ be the number of solitons and $N_{\bar{s}}$ the number of antisolitons in a scattering state of $\mathscr{H}_{\text {phys. }}$.

From the discussion above and from Lemma 8 and (61) we obtain

Corollary 5. (1) For every scattering state in the soliton-sectors $\mathscr{H}_{\pi}^{ \pm}, N_{s}-N_{\bar{s}}$ is odd.

(2) For every scattering state in the vacuum sectors $\mathscr{H}_{ \pm}, N_{s}-N_{\bar{s}}$ is even.

(3) Without taking into account solitons and anti-solitons, the scattering theory on $\mathscr{H}_{ \pm}$does not satisfy asymptotic completeness.

Remarks. Even if $\mathscr{H}_{\pi}^{ \pm}$do not contain one-( $\left.\begin{array}{c}\text { soliton } \\ \text { anti-soliton }\end{array}\right)$ states with discrete mass, we consider it as likely that $\mathscr{H}_{\text {phys. }}$ contains vectors describing resonances (the solitons and anti-solitons) of such a long life-time that they may undergo scattering.

A scattering theory for solitons of zero rest mass exists, as follows from the interesting results of [5]. The existence of solitons with zero rest mass can, however, presumably be excluded for $\sigma \gg 1$ on the basis of announced results [28].

The commutation relations (71) confirm that parity is spontaneously broken on $\mathscr{H}_{\pi}^{ \pm}$. Space reflection is the conjugation mapping $\mathscr{H}_{\pi}^{ \pm}$to $\mathscr{H}_{\pi}^{\mp} . \mathscr{H}_{\pi}^{+}$and $\mathscr{H}_{\pi}^{-}$ have opposite $\boldsymbol{Q}$-charge [where $\left.\boldsymbol{Q}=\int d x(\operatorname{grad} \phi)(x)\right]$.

\section{$\S 5$. The Solitons in the $P(\phi)_{2}$-Models}

Let $\phi$ be a neutral, scalar field in two space-time dimensions and let $P$ be an even polynomial with positive, leading coefficient. The quantum field models with interaction Lagrangian : $P(\phi)$ :, where the double colons denote Wick ordering with respect to some fixed bare mass, e.g. 1, are presently the best understood models of a relativistic quantum field theory; see [12, 36, 28, 40]. It has recently been proved that the $\phi \rightarrow-\phi$ symmetry of the Lagrangian is spontaneously broken by some pure vacuum states if the coefficient of the : $\phi^{2}$ :-term in $: P(\phi)$ : is sufficiently large and negative, [28], [at least if $P(\phi)=\lambda \phi^{4}-\sigma \phi^{2}$ ]. Under these circumstances we now show how to construct soliton- and anti-soliton sectors.

We want to emphasize here that the situation met in the analysis of the $P(\phi)_{2}$ models is quite different from the one in the $(\phi \cdot \phi)^{2}-\sigma \phi_{1}^{2}$ model, since in the former we are dealing with only one scalar, neutral field so that there is no obvious choice for $\mathrm{a}^{*}$-autormorphism $\varrho_{\alpha}$ with

$$
\varrho_{\alpha}(\phi(x))=\phi(x), \text { for } x<\underline{x}_{\alpha},
$$

and

$$
\varrho_{\alpha}(\phi(x))=-\phi(x), \text { for } \quad x>\bar{x}_{\alpha}>\underline{x}_{\alpha}
$$


and such that $\omega^{\circ} \varrho_{\alpha}$ is a space-time translation covariant soliton-state. Let $\omega_{+}$ be a $P(\phi)_{2}$ vacuum state with

$$
\omega_{+}(\phi(x, t))=\phi_{c}>0 \text {. }
$$

We let $\varrho_{\pi}$ be the *-automorphism determined by

$$
\varrho_{\pi}(\phi(x, t))=-\phi(x, t),
$$

and

$$
\omega_{-} \equiv \omega_{+}{ }^{\circ} \varrho_{\pi} .
$$

As in Sections $2,3\left(\mathscr{H}_{+}, \Omega_{+}\right)$and $\left(\mathscr{H}_{-}, \Omega_{-}\right)$denote the G.N.S. (झWightman-) Hilbert spaces and vacua reconstructed from $\omega_{+}, \omega_{-}$.

Definition 7. (tensor product $P(\phi)_{2}$-theory)

$$
\begin{aligned}
& \mathscr{H} \equiv \mathscr{H}_{+} \otimes \mathscr{H}_{-}, \omega(\cdot) \equiv\left\langle\Omega_{+} \otimes \Omega_{-}, \cdot \Omega_{+} \otimes \Omega_{-}\right\rangle \\
& \phi_{1} \equiv \phi \otimes I, \phi_{2} \equiv I \otimes \phi \quad \text { and } \quad \phi \equiv\left(\phi_{1}, \phi_{2}\right),
\end{aligned}
$$

where $\phi$ is the interacting $P(\phi)_{2}$ quantum field.

On the basis of this seemingly unnatural tensor product $P(\phi)_{2}$-theory [which we denote for short by $\left.P(\phi)_{2} \otimes P(\phi)_{2}\right]$ we may now construct soliton- and antisoliton states.

Let $\alpha^{+}$be a kink function satisfying the soliton-condition (see Definition 5). We define

$$
\begin{aligned}
& \varrho_{\alpha^{+}}\left(\phi_{1}(x)\right)=\cos \alpha^{+}(x) \cdot \phi_{1}(x)+\sin \alpha^{+}(x) \cdot \phi_{2}(x) \\
& \varrho_{\alpha^{+}}\left(\phi_{2}(x)\right)=-\sin \alpha^{+}(x) \cdot \phi_{1}(x)+\cos \alpha^{+}(x) \cdot \phi_{2}(x),
\end{aligned}
$$

plus identical equations for the corresponding canonically conjugate momenta; see also (17).

Let $\mathfrak{A}$ be the $\mathrm{C}^{*}$-algebra of all quasi-local observables generated by bounded functions of $\phi_{1}, \phi_{2}$ and their conjugate momenta; the precise definition is as in Section $2^{\prime}$. Let $\mathscr{H}_{\pi}^{ \pm}$be the G.N.S. Hilbert spaces associated with $\left(\omega^{\circ} \varrho_{\alpha^{ \pm}}, \mathfrak{U}\right)$, and $T_{\alpha^{ \pm}}$the isometry: $\mathscr{H} \rightarrow \mathscr{H}_{\pi}^{ \pm}$intertwining the representations of $\mathfrak{A}$ on $\mathscr{H}, \mathscr{H}_{\pi}^{ \pm}$, respectively.

With these definitions Theorem 3 remains true for the $P(\phi)_{2} \otimes P(\phi)_{2}$-theory, and the proof is almost verbally the same as the one in the case of the $(\phi \cdot \phi)_{2}^{2}$ theory. (We notice that for the $P(\phi)_{2} \otimes P(\phi)_{2}$-theory on the vacuum sector $\mathscr{H}$ all Wightman axioms are known $[19,36]$ and that the cluster properties (49) - see Lemma 6 - hold in almost all pure phases [19].)

The spatial cluster properties imply that the vacuum $\Omega_{+} \otimes \Omega_{-}$is unique. From this and results of [2] we conclude that the weak closure of $\mathfrak{U}_{\text {on }} \mathscr{H}_{+} \otimes \mathscr{H}_{-}$ coincides with $B\left(\mathscr{H}_{+} \otimes \mathscr{H}_{-}\right)$. As in Section 3 it then follows that the weak closure of $\mathscr{U}$ on $\mathscr{H}_{\pi}^{ \pm}$is equal to $B\left(\mathscr{H}_{\pi}^{ \pm}\right)$. By the $P(\phi)_{2} \otimes P(\phi)_{2}$ version of Theorem 3 the space-time translation automorphisms of $\mathfrak{U}$ are implemented on $\mathscr{H}_{\pi}^{ \pm}$by a unitary group

$$
\left\{e^{i\left(t H_{\pi}^{ \pm}-x P^{ \pm}\right)} /(t, x) \in \mathbb{R}^{2}\right\}
$$

which satisfies the relativistic spectrum condition. 
We now construct a soliton-state for the $P(\phi)_{2}$-theory which is a factor state.

Let $\mathfrak{A}_{1}$, be the $C^{*}$-algebra of all quasi-local observable generated by bounded functions of $\phi_{1}$ and $\pi_{1}$; see Section $2^{\prime}$. Let $\mathfrak{U}_{1}^{ \pm}$denote its weak closure on $\mathscr{H}_{\pi}^{ \pm}$. Since the weak closure of $\mathfrak{U}$ on $\mathscr{H}_{\pi}^{ \pm}$is a factor of type $I_{\infty} \mathfrak{U}_{1}^{ \pm}$is a factor, as well.

For $A \in \mathfrak{U}_{1}^{ \pm}$

$$
\tau_{\xi}(A) \equiv e^{i\left(t H_{\pi}^{ \pm}-x P_{\pi}^{ \pm}\right)} A e^{-i\left(t H_{\pi}^{ \pm}-x P_{\pi}^{ \pm}\right)} \in \mathfrak{U}_{1}^{ \pm} .
$$

By a theorem of Borchers [7] there exists a continuous, unitary group $\left\{e^{i\left(t H_{\pi, 1}^{ \pm}-x P_{\pi, 1}^{ \pm}\right)} / \xi \in \mathbb{R}^{2}\right\} \subset \mathfrak{U}_{1}^{ \pm}$which satisfies the relativistic spectrum condition and implements $\tau_{\xi} \uparrow \mathfrak{U}_{1}$.

Thus $\omega_{ \pm} \uparrow \mathfrak{U}_{1}$ is a space-time translation covariant factor state and, by construction, it is a soliton-state for the $P(\phi)_{2}$-theory.

We conjecture that $\mathfrak{U}_{1}^{ \pm}$is a factor of type $I_{\infty}$. If this is true there exists a tensor product decomposition

$$
\begin{aligned}
\mathscr{H}_{\pi}^{ \pm} & =\mathscr{H}_{\pi, 1}^{ \pm} \otimes \mathscr{H}_{\pi, 2}^{ \pm} \\
\mathfrak{U}_{1}^{ \pm} & =B\left(\mathscr{H}_{\pi, 1}^{ \pm}\right) \otimes I \\
e^{i\left(t H_{\pi, 1}^{ \pm}-x P_{\pi, 1}^{ \pm}\right)} & =e^{i\left(t H_{\pi, 1}^{ \pm}-x P_{\pi, 1}^{ \pm}\right)} \uparrow \mathscr{H}_{\pi, 1}^{ \pm} \otimes I \nmid \mathscr{H}_{\pi, 2}^{ \pm} .
\end{aligned}
$$

We can then drop the trivial factor $\mathscr{H}_{\pi, 2}^{ \pm}$. The vectors in $\mathscr{H}_{\pi, 1}^{ \pm}$are pure, space-time translation covariant soliton-states, for the $P(\phi)_{2}$-theory. Let $\Pi_{\mathscr{H}^{ \pm}, 1}, \Pi_{\mathscr{H}}+$ denote the representation of $\mathfrak{U}_{1}$ on $\mathscr{H}_{\pi, 1}^{ \pm}, \mathscr{H}_{+}$, respectively. Still assuming that our conjecture is true one can show, using a theorem of [43], that

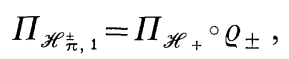

for some ${ }^{*}$-automorphism $\varrho_{ \pm}$of $\mathfrak{U}_{1}$.

With $\left(\mathfrak{U}_{1}, \mathscr{H}_{\pi}^{ \pm},\left(H_{\pi, 1}^{ \pm}, P_{\pi, 1}^{ \pm}\right)\right)\left[\left(\mathfrak{U}_{1}, \mathscr{H}_{\pi, 1}^{ \pm},\left(H_{\pi, 1}^{ \pm}, P_{\pi, 1}^{ \pm}\right)\right)\right.$, resp. $]$replacing $\left(\mathfrak{A}, \mathscr{H}_{\pi}^{ \pm}\right.$, $\left.\left(H_{\pi}^{ \pm}, P_{\pi}^{ \pm}\right)\right)$Theorem 4 remains literally true for the $P(\phi)_{2}$-model. Parity is spontaneously broken on the soliton sectors $\mathscr{H}_{\pi,(1)}^{ \pm}$. The analysis of Section 4 applies to this case, as well. It follows from announced results of Glimm et al. [28], that for $\sigma \gg 1$ the spectrum of $(H, P)$ on $\mathscr{H}_{+}$and $\mathscr{H}_{-}$has a mass gap which excludes soliton-states of rest mass 0 !

Concluding remarks: By considering the $P(\phi)_{2} \otimes P(\phi)_{2}$-theory we are able to construct soliton-states for the $P(\phi)_{2}$-theory that are factor states, and presumably they are pure states of the form $\omega_{+}{ }^{\circ} \varrho_{ \pm}$, for some $*_{\text {-automorphism }}$ $\varrho_{ \pm}$of $\mathfrak{U}_{1}$.

By using a different Bogoliubov transformation we can easily construct pure $P(\phi)_{2}$-states with non-vanishing charge $Q=\int d x(\operatorname{grad} \phi)(x)$. However these states are presumably not space-time translation covariant. For the $\left(\lambda \phi^{4}\right)_{2}$-model on a space lattice in the two phase region we can prove existence of pure, charged space-time translation covariant soliton-states.

\section{§ 6. Soliton-Sectors and Soliton-Automorphisms: An Outline of a General Theory}

In this section we draw abstract conclusions from what we learnt in the previous sections about soliton-sectors by studying specific models. The setup we are going to use must be placed in the framework of algebraic quantum field theory $[9,6]$. 
According to our personal taste we would like to present the following general results within the framework of local morphisms on the algebra of all local observables [9]. This would, however, go beyond the purposes of this paper. Therefore we rather make the framework of [9] somewhat more concrete so that the typical properties found for the soliton-states in the models are still present in this general setup and the peculiarities of two space-time dimensions remain apparent. We remark, however, that most of the following results can be proved in the more general situation considered in [9]. Some of them are scattered about different places of [9]. We hope to present a summary of such results elsewhere. In the following a hypothesis which becomes a theorem for the models considered in Sections $1-5$ is marked by $(*)$.

Let $\left(\mathfrak{A}, \tau_{\xi}, \omega\right)$ be an algebra of local observables, a space-time translation automorphism group on $\mathfrak{U}$ and a state on $\mathfrak{U}$ which is invariant under $\left\{\tau_{\xi}\right\} \equiv$ $\left\{\tau_{\xi} \mid \xi \in \mathbb{R}^{2}, \xi \equiv(x, t)\right\}$.

The G.N.S. Hilbert space, the cyclic vector, the scalar product and the unitary space-time translation group implementing $\left\{\tau_{\xi}\right\}$ reconstructed from $\left(\mathfrak{U}, \tau_{\xi}, \omega\right)$ are denoted by $\mathscr{H}, \Omega,\langle\cdot, \cdot\rangle$ and $\left\{T_{\xi}\right\}$ respectively.

We assume that $\mathscr{H}$ is a separable Hilbert space and $\left\{T_{\xi}\right\}$ satisfies the relativistic spectrum condition. In this case $\omega$ is called a vacuum state and $\Omega$ a vacuum. Throughout the following we may assume that $\omega$ is a pure vacuum state so that the representation of $\mathfrak{U}$ on $\mathscr{H}$ is irreducible [2]. Let $\mathscr{F}$ be the family of all compact diamonds in $\mathbb{R}^{2}$ with non-empty interior. We suppose that

$$
\mathfrak{A}=\overline{\bigcup_{\mathscr{U} \in \mathscr{F}} \mathfrak{A}(\mathcal{O})} \text { norm, }
$$

where $\{\mathfrak{U}(\mathcal{O}) \mid \mathcal{O} \in \mathscr{F}\}$ is a family of local von Neumann algebras with the properties:

1) If $\mathcal{O}_{1}$ and $\mathcal{O}_{2}$ are in $\mathscr{F}$

$$
\mathfrak{U}\left(\mathcal{O}_{1}\right) \wedge \mathfrak{U}\left(\mathcal{O}_{2}\right)=\mathfrak{U}\left(\mathcal{O}_{1} \cap \mathcal{O}_{2}\right) ;
$$

Merely as a matter of convenience we also assume that for $\mathcal{O} \in \mathscr{F}, \mathfrak{A}(\mathcal{O})$ is a factor. (Since $\omega$ is pure, such an assumption is possible.)

If $\hat{\mathcal{O}}$ is an unbounded region, e. g. a cone, we define

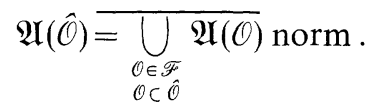

If $\mathfrak{B}$ is an algebra of operators on $\mathscr{H}, \mathfrak{B}^{\prime}$ denotes its commutant and $\mathfrak{B}^{\prime \prime}$ its weak closure.

Given any diamond or cone $\mathcal{O}$ we define

$\mathcal{O}_{x}=\{\xi \mid \xi-(x, 0) \in \mathcal{O}\}$

$\mathcal{O}_{t}:$ the smallest diamond containing all points

$\{\xi \equiv(x, s) \mid \exists(y, s) \in \mathcal{O} \quad$ with $|x-y| \leqq|t|\}$

$\mathcal{O}_{\xi}=\left(\mathcal{O}_{x}\right)_{t}$, with $\xi=(x, t)$

$\sim \mathcal{O}$ : causal complement of $\mathcal{O}$.

We notice that for $\mathcal{O} \in \mathscr{F}, \sim \mathcal{O}=\mathcal{O}_{L} \cup \mathcal{O}_{R}$, where $\mathcal{O}_{L}$ and $\mathcal{O}_{R}$ are two disjoint cones opening to the left, the right, respectively. 
If two regions $\mathcal{O}_{1}, \mathcal{O}_{2}$ are space-like separated, we write $\mathcal{O}_{1} \times \mathcal{O}_{2}$. The standard locality hypothesis is: For $\mathcal{O} \in \mathscr{F}, \mathfrak{U}(\mathcal{O}) \subseteq \mathfrak{U}(\sim \mathcal{O})^{\prime}$.

We assume furthermore:

2) Duality: For $\mathcal{O} \in \mathscr{F}$

$$
\mathfrak{U}(\mathcal{O})^{\prime}=\mathfrak{U}(\sim \mathcal{O})^{\prime \prime}
$$

See [6] for the problems connected with locality and duality and for the construction of local algebras satisfying duality $(*)$.

3) Finite Propagation Speed: If $\mathcal{O}$ is a diamond or a cone,

$$
\tau_{\xi}(\mathfrak{U}(\mathcal{O})) \cong \mathfrak{U}\left(\mathcal{O}_{\xi}\right) .
$$

We let $G$ be the class $\left\{\varrho_{\chi} \mid \chi \in X, X\right.$ some index set $\}$ of local *automorphisms on $\mathfrak{U}$ such that

$$
\begin{aligned}
& \varrho_{\chi}(\mathfrak{U}(\mathcal{O})) \subseteq \mathfrak{U}(\mathcal{O}), \quad \text { for all diamonds (cones) } \mathcal{O}, \\
& \tau_{\xi^{\circ}} \varrho_{\chi}(A)=\varrho_{\chi} \tau_{\xi}(A), \quad \text { for all } A \in \mathfrak{U} .
\end{aligned}
$$

Clearly all states $\left\{\omega^{\circ} \varrho_{\chi} \mid \chi \in X\right\}$ are space-time translation invariant, and $G$ is a group; $X$ becomes a group if we associate with $\chi_{1}, \chi_{2}$ the element $\chi_{1} \cdot \chi_{2} \in X$ with the property that $\varrho_{\chi_{1}} \varrho_{\chi_{2}}=\varrho_{\chi_{1} \cdot \chi_{2}}$. Then $\chi_{1} \cdot \chi_{2}$ is uniquely determined: All states $\left\{\omega^{\circ} \varrho_{\chi} \mid \chi \in X\right\}$ satisfy the cluster property - see (49) - , since $\omega$ is pure and by (76). Thus if $\omega^{\circ} \varrho_{\chi^{\prime}}$ is a vector state of $\omega^{\circ} \varrho_{\chi}$ then $\varrho_{\chi^{\prime}}=\varrho_{\chi}$. The group $G$ is a representation of $X$ on $\mathfrak{A}$.

We let $e$ be the identity of $X$, i. e. $\varrho_{e}(A)=A$, all $A \in \mathfrak{A}$. By (76) $X$ is a symmetry group of the dynamics. If $X \supsetneqq\{e\}, \omega$ breaks the symmetry $X$.

Definition 8 . We denote by $\sigma_{e-\chi}$ any automorphism of $\mathfrak{A}$ with the property that there exists $\mathcal{O} \in \mathscr{F}$ such that

$$
\left.\begin{array}{l}
\sigma_{e-\chi}(A)=A, \text { for all } A \in \mathfrak{U}\left(\mathcal{O}_{L}\right), \text { and } \\
\sigma_{e-\chi}(A)=\varrho_{\chi}(A), \text { for all } A \in \mathfrak{U}\left(\mathcal{O}_{R}\right) ;
\end{array}\right\}
$$

$\operatorname{supp} \sigma_{e-\chi}$ is the smallest diamond $\mathcal{O}$ for which (77) holds; $\sigma_{\chi-e}$ and supp $\sigma_{\chi-e}$ are defined by exchanging the roles of $L$ and $R$. Finally $\sigma_{\chi}=\sigma_{e-\chi}$ or $\sigma_{\chi-e}$.

4) Existence of the Automorphisms $\sigma_{\chi},(*)$ : Given arbitrary $\chi \in X$ and $\mathcal{O} \in \mathscr{F}$ we assume that automorphisms $\sigma_{\chi}$ satisfying (77) with $\operatorname{supp} \sigma_{\chi}=\mathcal{O}$ exist.

So far we have not imposed specific properties on the automorphisms $\sigma_{\chi}$ which distinguish whether $\omega^{\circ} \sigma_{\chi}$ is a soliton - or a non-translation invariant vacuum state.

5) Equivalence (*): If $\sigma_{\chi}^{1}$ and $\sigma_{\chi}^{2}$ are two automorphisms satisfying (77), then $\omega \circ \sigma_{\chi}^{1}$ is a vector state of $\omega^{\circ} \sigma_{\chi}^{2}$, i. e. $\sigma_{\chi}^{1} \circ\left(\sigma_{\chi}^{2}\right)^{-1}$ is unitarily implementable on $\mathscr{H}$.

Remark. If the algebraic theory defined by $\left(\mathfrak{A}, \tau_{\xi}, \omega\right)$ is derived from the theory of finitely many canonical Bose fields and $\mathfrak{A}(\mathcal{O})$ is *-isomorphic to $\mathfrak{A}_{F}(\mathcal{O})$, the corresponding local free field algebra, for $\mathcal{O} \in \mathscr{F}$, then 4) and 5) are theorems. The proof follows by abstraction of our construction in Section 5 and Lemmata 1, 2 of Section 3. 
Definition 9. An automorphism $\sigma_{\chi}$ satisfying 4) and 5) is called covariant (or a "soliton"-automorphism) if the space-time translation automorphisms $\left\{\tau_{\xi}\right\}$ on $\mathfrak{A}$ are unitarily implementable on the G.N.S. Hilbert space $\mathscr{H}_{\chi}$ reconstructed from $\left(\omega^{\circ} \sigma_{\chi}, \mathfrak{U}\right)$. The corresponding unitary group on $\mathscr{H}_{\chi}$ is denoted by $\left\{T_{\xi}^{\chi}\right\}$. If $\sigma_{\chi}$ is a covariant automorphism, there exists a unitary group $\left\{T_{\xi}^{\sigma_{\chi}}\right\}$ on $\mathscr{H}$ such that $\left\langle\Omega,\left(T_{\xi}^{\sigma}\right)^{*} \sigma_{\chi}(A) T_{\xi}^{\sigma_{\chi}} \Omega\right\rangle=\omega \circ \sigma_{\chi}\left(\tau_{\xi}(A)\right)$.

We define the cocycles

$$
V_{\xi}^{\sigma_{\chi}} \equiv T_{\xi}^{\sigma_{\chi}} T_{\xi}^{*} \text {. }
$$

Lemma 9. Assume that 1)-5) hold. Let $\sigma_{\chi}$ be covariant. Then

(1) $V_{\xi_{1}+\xi_{2}}^{\sigma_{\chi}}=V_{\xi_{1}}^{\sigma_{\chi}} \tau_{\xi_{1}}\left(V_{\xi_{2}}^{\sigma_{\chi}}\right)$

(2) $V_{\xi}^{\sigma_{\chi}} \in \mathfrak{U}\left(\left(\operatorname{supp} \sigma_{\chi}\right)_{\xi}\right)$.

Proof. (1) is an immediate consequence of (78).

(2) Let $A \in \mathfrak{A}\left(\sim\left(\operatorname{supp} \sigma_{\chi}\right)_{\xi}\right)$. Then

$$
\begin{aligned}
& V_{\xi}^{\sigma_{\chi}} A\left(V_{\xi}^{\sigma_{\chi}}\right)^{*}=T_{\xi}^{\sigma_{\chi}} \tau_{\xi}(A)\left(T_{\xi}^{\sigma_{\chi}}\right)^{*} \\
& =T_{\xi}^{\sigma_{\chi}} \sigma_{\chi} \circ \sigma_{\chi}^{-1} \circ \tau_{\xi}(A)\left(T_{\xi}^{\sigma \chi}\right)^{*}=\sigma_{\chi}^{\circ} \tau_{-\xi^{\circ}} \sigma_{\chi}^{-1} \circ \tau_{\xi}(A) \\
& =\left\{\begin{array}{l}
\sigma_{\chi}(A), \quad \text { if } \tau_{\xi}(A) \in \mathfrak{A}\left(\left(\operatorname{supp} \sigma_{\chi}\right)_{L}\right) \\
\sigma_{\chi} \circ \tau_{-\xi^{\circ}} \varrho_{\chi}^{-1} \circ \tau_{\xi}(A), \quad \text { if } \tau_{\xi}(A) \in \mathfrak{U}\left(\left(\operatorname{supp} \sigma_{\chi}\right)_{R}\right)
\end{array}\right. \\
& =A \text {, by (76) and (77). }
\end{aligned}
$$

The proof is completed by using assumption 2).

Given $\sigma_{\chi}$ we set

Q.E.D.

$$
\sigma_{\chi, x}=\tau(x, 0) \circ \sigma_{\chi} \circ \tau_{(-x, 0)}
$$

By 5) $\sigma_{\chi, x}$ and $\sigma_{\chi}$ are equivalent, and $\sigma_{\chi}^{x} \equiv \sigma_{\chi}{ }^{\circ} \sigma_{\chi, x}^{-1}$ is unitarily implemented on $\mathscr{H}$. If $\sigma_{\chi}$ is covariant and $\left(\operatorname{supp} \sigma_{\chi}\right)_{\xi} \times\left(\operatorname{supp} \sigma_{\chi, x}\right)$, then $V_{\xi}^{\sigma_{\chi}^{x}}=V_{\xi}^{\sigma_{\chi}} V_{\xi}^{\sigma_{x}, x},\left({ }^{*}\right)$ : By 1), 2) and the Lemma, $V_{\xi}^{\sigma_{x}^{x}}$ is in $\mathfrak{A}\left(\operatorname{supp} \sigma_{\chi}\right) \vee \mathfrak{A}\left(\operatorname{supp} \sigma_{\chi, x}\right)$ and so is $V_{\xi}^{\sigma_{\chi}} V_{\xi}^{\sigma_{x}, x}$. As in the proof of part (2) of Lemma 9, one shows that $V_{\xi}^{\sigma_{\chi}^{x}}\left(V_{\xi}^{\sigma_{\chi}} V_{\xi}^{\sigma_{\chi}, x}\right)^{*} \in\left(\mathfrak{A}\left(\operatorname{supp} \sigma_{\chi}\right) \vee\right.$ $\left.\mathfrak{U}\left(\operatorname{supp} \sigma_{\chi, x}\right)\right)^{\prime}$. If we choose $T_{\xi}^{\sigma_{\chi}}$ and $T_{\xi}^{\sigma_{x, x}}$ properly and apply 1$)$ we conclude that $V_{\xi}^{\sigma x}\left(V_{\xi}^{\sigma x, x} V_{\xi}^{\sigma x, x}\right)^{*}=I$.

Theorem 6. Assume 1)-5).

Then the following are equivalent:

(1) $\sigma_{\chi}$ is covariant.

(2) If $\left(\operatorname{supp} \sigma_{\chi}\right)_{\xi} \times\left(\operatorname{supp} \sigma_{\chi, x}\right)_{\xi}$.

$V_{\xi}^{\sigma x}=V_{\xi}^{1} V_{\xi}^{2}$, with $V_{\xi}^{1}, V_{\xi}^{2}$ weakly measurable in $\xi$ on $\mathscr{H}$ and $V_{\xi}^{1} \in \mathfrak{A}\left(\left(\operatorname{supp} \sigma_{\chi}\right)_{\xi}\right)$, $V_{\xi}^{2} \in \mathfrak{U}\left(\left(\operatorname{supp} \sigma_{\chi, x}\right)_{\xi}\right),\left(^{*}\right)$.

Proof. $(1) \Rightarrow(2)$ is already proved.

$(2) \Rightarrow(1)$ : Since by 5) $\sigma_{\chi}^{x}$ is unitarily implemented on $\mathscr{H}$,

$V_{\xi}^{\sigma_{x}^{x}} T_{\xi}=\sigma_{\chi}^{x}\left(T_{\xi}\right)$ is a strongly continuous unitary group on $\mathscr{H}$ satisfying the relativistic spectrum condition. 
From (79) and 1) it follows that $V_{\xi}^{1}$ and $V_{\xi}^{2}$ satisfy the cocycle identity (1) of Lemma 9, provided $\xi_{1}$ and $\xi_{2}$ are so small that

$$
\left(\operatorname{supp} \sigma_{\chi}\right)_{\xi_{1}+\xi_{2}} \times\left(\operatorname{supp} \sigma_{\chi, x}\right)_{\xi_{1}+\xi_{2}} .
$$

Hence for $|\xi|$ sufficiently small $V_{\xi}^{1} T_{\xi}$ and $V_{\xi}^{2} T_{\xi}$ are weakly measurable, unitary groups and hence strongly continuous, since $\mathscr{H}$ is separable. By letting $x$ tend to $\infty$ we complete the proof by the arguments used to prove Lemma 7, Section 3 .

Q.E.D.

Theorem 7. If $\sigma_{\chi}$ is covariant then $\left\{T_{\xi}^{\chi}\right\}$ satisfies the relativistic spectrum condition, and the spectrum of the space-translations is purely continuous. The assertions of Theorems 3 and 4 remain true in this general context.

Proof. The spectrum condition is derived as in Section 3, Lemma 7, (3). Assuming covariance of the soliton state, the proofs for the remaining assertions of Theorems 3 and 4 are model independent.

Q.E.D.

Theorem 8. The equivalence classes of covariant automorphisms - see 5) form a group (which we call the "soliton group").

Proof. Let $\sigma_{\chi_{1}}, \sigma_{\chi_{2}}$ be covariant. By 5) we may assume that for $|\xi| \leqq 1$

$$
\left(\operatorname{supp} \sigma_{\chi_{2}}\right)_{\xi} \subset\left(\operatorname{supp} \sigma_{\chi_{1}}\right)_{L} \text {. }
$$

We claim that for $|\xi| \leqq 1$

$$
V_{\xi}^{\sigma} x_{2} V_{\xi}^{\sigma \chi_{1}} T_{\xi} \equiv T_{\xi}^{\sigma \chi_{2} \cdot x_{1}}
$$

satisfies:

$$
\omega \circ \sigma_{\chi_{1}} \circ \sigma_{\chi_{2}}\left(\tau_{\xi}(A)\right)=\left\langle\Omega,\left(T_{\xi}^{\sigma} \chi_{1} \cdot \chi_{2}\right)^{*} \sigma_{\chi_{1}} \circ \sigma_{\chi_{2}}(A) T_{\xi}^{\sigma} \chi_{\chi_{1}} \cdot \chi_{2} \Omega\right\rangle,
$$

for all $A \in \mathfrak{U}$.

It suffices to prove this for $A \in \mathfrak{A}(\mathcal{O})$, any $\mathcal{O} \in \mathscr{F}$.

$$
\begin{aligned}
& \left\langle\Omega,\left(T_{\xi}^{\sigma \chi_{1} \cdot \chi_{2}}\right)^{*} \sigma_{\chi_{1}}{ }^{\circ} \sigma_{\chi_{2}}(A) T_{\xi}^{\sigma \chi_{1} \cdot \chi_{2}} \Omega\right\rangle \\
& =\left\langle\Omega,\left(V_{\xi}^{\sigma \chi_{1}}\right)^{*}\left(V_{\xi}^{\sigma \chi_{2}}\right)^{*} \sigma_{\chi_{1}}{ }^{\circ} \sigma_{\chi_{2}}(A) V_{\xi}^{\sigma \chi_{2}} V_{\xi}^{\sigma \chi_{1}} \Omega\right\rangle \\
& =\left\langle\Omega,\left(V_{\xi}^{\sigma \chi_{1}}\right)^{*} \sigma_{\chi_{1}}\left(\left(V_{\xi}^{\sigma \chi_{2}}\right)^{*} \sigma_{\chi_{2}}(A) V_{\xi}^{\sigma \chi_{2}}\right) V_{\xi}^{\sigma \chi_{1}} \Omega\right\rangle,
\end{aligned}
$$

by (80), (77) and Lemma 9 ,

$$
\begin{aligned}
& =\left\langle\Omega, \sigma_{\chi_{1}}\left(\tau_{\xi}\left(\left(V_{\xi}^{\sigma} \chi_{2}\right)^{*} \sigma_{\chi_{2}}(A) V_{\xi}^{\sigma} \chi_{2}\right)\right) \Omega\right\rangle \\
& =\left\langle\Omega, \sigma_{\chi_{1}}{ }^{\circ} \sigma_{\chi_{2}}\left(\tau_{\xi}(A)\right) \Omega\right\rangle .
\end{aligned}
$$

Proof of (81). For all $\Psi \in \mathscr{H}$

$$
\begin{aligned}
\sigma_{\chi_{2}}\left(\tau_{\xi}(A)\right) \Psi & =T_{\xi}^{*}\left(V_{\xi}^{\sigma} \chi_{2}\right)^{*} \sigma_{\chi_{2}}(A) V_{\xi}^{\sigma \chi_{2}} T_{\xi} \Psi \\
& =\tau_{\xi}\left(\left(V_{\xi}^{\sigma \chi_{2}}\right)^{*} \sigma_{\chi_{2}}(A) V_{\xi}^{\sigma_{\chi_{2}}}\right) \Psi .
\end{aligned}
$$

Since $A \in \mathfrak{A}(\mathcal{O})$, some $\mathcal{O} \in \mathscr{F}$, and by Lemma 9 there exists $\hat{\mathcal{O}} \in \mathscr{F}$ such that $\tau_{\xi}\left(\left(V_{\xi}^{\sigma} \chi_{2}\right)^{*}\right.$ $\left.\sigma_{\chi_{2}}(A) V_{\xi}^{\sigma_{\chi_{2}}}\right)$ and $\sigma_{\chi_{2}}\left(\tau_{\xi}(A)\right)$ are in $\mathfrak{U}(\hat{\mathcal{O}})$. 
By 3$)$ and the relativistic spectrum condition, $\mathscr{H}$ contains a separating vector for $\mathfrak{A}(\hat{\mathcal{O}})$. Therefore

$$
\sigma_{\chi_{2}}\left(\tau_{\xi}(A)\right)=\tau_{\xi}\left(\left(V_{\xi}^{\sigma \chi_{2}}\right)^{*} \sigma_{\chi_{2}}(A) V_{\xi}^{\sigma} \chi_{2}\right)
$$

which completes the proof of (81).

Finally, using the group property, we may extend $\left\{T_{\xi}^{\sigma \chi_{1} \cdot x_{2}} \| \xi \mid \leqq 1\right\}$ to all $\xi \in \mathbb{R}^{2}$.

Q.E.D.

Corollary 9. Assume 1)-5).

(1) If $X$ is abelian, the soliton group is abelian.

(2) All states $\left\{\omega^{\circ} \varrho_{\chi} \mid \chi \in X\right\}$ are vacuum states, (i.e. the relativistic spectrum condition is satisfied).

Proof.(1) follows directly from (77) and 5), (i.e. "Equivalence").

(2) $\omega \circ \varrho_{\chi}=\lim _{x \rightarrow-\infty} \omega \circ \sigma_{e-\chi, x}$.

Using Theorem 7 and the arguments in the proof of Lemma 7, (3), Section 3, the corollary follows.

Q.E.D.

Examples of Soliton-Groups. For the models considered in Sections 3-5 we obtain the following abelian soliton group:

Set $e \equiv \varrho_{e}, s \equiv \varrho_{\alpha^{+}}, \bar{s} \equiv \varrho_{\alpha^{-}}$, and $i \equiv \varrho_{\alpha^{+}}{ }^{\circ} \varrho_{\alpha^{-}}$. Then the multiplication table of the soliton group is given by

\begin{tabular}{|c|c|c|c|c|}
\hline & $e$ & $s$ & $\bar{s}$ & $i$ \\
\hline$e$ & $e$ & $s$ & $\bar{s}$ & $i$ \\
\hline$s$ & $s$ & $e$ & $i$ & $\bar{s}$ \\
\hline $\bar{s}$ & $\bar{s}$ & $i$ & $e$ & $s$ \\
\hline$i$ & $i$ & $\bar{s}$ & $s$ & $e$ \\
\hline
\end{tabular}

For the $\cos \varepsilon \phi_{2}$ theory (see Sections $1,1^{\prime}$ ) the soliton-group is $\mathbb{Z}$. A more detailed analysis of the soliton-states for the $\cos \varepsilon \phi_{2}$-model and a class of related models is of considerable pedagogical value for the understanding of the general theory outlined in this section. It appears in a forthcoming paper, [15].

Remarks. 1) It is easy to conceive two dimensional models with a non-abelian soliton group. Consider e.g. a scalar Bose field $\phi=\left(\phi_{1}, \ldots, \phi_{n}\right), n \geqq 3$, with selfinteractions given by

$$
\mathscr{S}_{I}=g:(\boldsymbol{\phi} \cdot \boldsymbol{\phi})^{2}:_{m}-\frac{3}{2} g \sum_{1 \leqq i<j \leqq n}: \phi_{i}^{2} \phi_{j}^{2}:_{m}
$$

with $m^{2} / g \ll 1$. Assuming existence and $\phi_{i} \rightarrow-\phi_{i}$ symmetry breaking, $i=1, \ldots, n$, this model has a non-abelian soliton-group. It follows from the Goldstone 
theorem [13] that in two dimensions neither $X$ nor the soliton group can be continuous Lie groups but are typically (subgroups of the) permutation groups.

2) As a heuristic principle we derive from our analysis that an automorphism $\sigma_{\chi}$ satisfying 4) and 5) is covariant if and only if the cocycle $V_{\xi}^{\sigma_{\chi}}$ is a local observable.

If extended to arbitrarily many space-time dimensions this principle tells us that our constructions in Sections 1-6 ought to break down in three or more space-time dimensions. If $\sigma_{\chi}$ is a (soliton-) automorphism of the kind introduced in Definition 8 we define $\operatorname{supp} \sigma_{\chi}$ to be the intersection of all diamonds $\mathcal{O}$ with the property that for $A \in \mathfrak{A}(\sim \mathcal{O})$ there exists a positive number $r(A)$ such that

$$
\tau_{\xi} \circ \sigma_{\chi}(A)=\sigma_{\chi} \circ \tau_{\xi}(A), \text { for all }|\xi|<r(A) .
$$

In three or more space-time dimensions $\operatorname{supp} \sigma_{\chi}$ always contains an unbounded diamond, provided $\sigma_{\chi}$ is not unitarily implementable and the theory has no gauge symmetry. Hence - if it existed - the cocycle $V_{\xi}^{\sigma_{x}}$ would not be a local observable. In a physicists language: The state $\omega^{\circ} \sigma_{\chi}$ has infinite energy with probability 1 . Therefore, by our principle, $\sigma_{\chi}$ is not covariant.

3) This situation changes if we consider local morphisms [9] or gauge transformations. In this case we may assume that there exists a compact diamond (1) such that

$$
\sigma_{\chi}(A)=A,
$$

and

$$
\tau_{\xi^{\circ}} \sigma_{\chi}(A)=\sigma_{\chi} \circ \tau_{\xi}(A), \text { for all } A \in \mathfrak{A}(\sim \mathcal{O}),
$$

$\sigma_{\chi}$ is not unitarily implementable on $\mathscr{H}$.

If we consider the action of $\sigma_{\chi}$ on unobservable (charged) field operators localized in $\sim \mathcal{O}$ then (82) and (83) imply that $\sigma_{\chi}$ is just a (spacedependent) gauge transformation. Therefore we call $\sigma_{\chi}$ a gauge (auto-) morphism.

If the gauge invariance of the theory is dynamically broken in the state $\omega$ then there may exist gauge automorphisms $\sigma_{\chi}$ satisfying (82), (83) which also satisfy (84). They are accompanied by "topological quantum numbers," $[14,35]$ and refs. given there. A field theory constituting a model for this situation in four space-time dimensions is necessarily a non-abelian Yang-Mills theory. We would e.g. study a scalar pion field interacting with an SU(2) Yang-Mills field. We summarize our speculations as follows:

$\left.\begin{array}{l}\text { Theory for which gauge } \\ \text { automorphisms satisfying } \\ \text { (82)-(84) exist }\end{array}\right\} \Rightarrow\left\{\begin{array}{l}\text { Theory is a Yang-Mills theory, } \\ \text { gauge invariance is dynamically } \\ \text { broken in the vacuum sector; } \\ \text { occurrence of "topological } \\ \text { quantum numbers". }\end{array}\right.$

4) In more than two space-time dimensions there may exist automorphisms $\sigma_{\chi}$ satisfying Definition 8, (77) which have, however, the property that $\omega^{\circ} \sigma_{\chi}$ 
is time-translation invariant. The state $\omega \circ \sigma_{\chi}$ should then not be called a solitonstate. It is rather a non-translation invariant vacuum state. Such states are known to exist in the three (or more) dimensional Ising model.

In the case of the $\phi^{4}$ quantum field model non-translation invariant vacuum states may exist in three or more space-time dimensions: The field equation

$$
\left(\square+m^{2}\right) \phi(\underline{x}, t)=-\lambda \phi(\underline{x}, t)^{3}
$$

has the solution

$$
\phi_{c}\left(\underline{x}^{1}\right)=(m / \sqrt{\lambda}) \tanh \left(m x^{1} / \sqrt{\lambda}\right), \quad[35] .
$$

Assuming that $\lambda$ and $m$ are chosen such that we are in the two phase region we may then introduce a new quantum field

$$
\phi^{\prime}(\underline{x}, t)=\phi(\underline{x}, t)-\phi_{c}\left(\underline{x}^{1}\right)
$$

and compute the $\phi^{4}$-Hamiltonian as a functional of $\phi^{\prime}$, [35]. It turns out that the substitution (85) introduces a space-dependent bare mass term in the Hamiltonian density which is very large for $\left|x_{1}\right| \gg 1$. Therefore a cluster expansion of the kind announced in [28] ought to be a suitable tool for the construction of a vacuum sector for the Hamiltonian expressed as a functional of $\phi^{\prime}$ and the field $\phi^{\prime}$. The vacuum expectation value $\langle\phi(x, t)\rangle$ would then resemble $\phi_{c}$, would, however, be modified by quantum corrections; it is independent of $t$. (Compare this to Section 5.)

5) One might conjecture that the spontaneous breaking of a symmetry in a quantum field model in more than one space dimension is usually accompanied by the formation of new bound states: "Bound states" of two or more "would be solitons" which are confined, by the arguments of Remark 2. A verification of this conjecture might be of interest to the problem of confinement.

The phenomenon discussed here should also occur in one space dimension in theories like $\left[g(\boldsymbol{\phi} \cdot \boldsymbol{\phi})^{2}-\sigma \phi_{1}^{2}-\mu \phi_{1}\right]_{2}$ : Choose $g$ and $\sigma$ such that for $\mu=0$ the $\phi_{1} \mapsto-\phi_{1}$ symmetry is spontaneously broken and there exists soliton-sectors. Then for small $|\mu| \neq 0$ this model is expected to have a rich particle spectrum of bound states of two "would be solitons". At present there is no rigorous proof for this conjecture.

6) The author has recently proven that the Lorentz automorphisms are unitarily implemented on the soliton-sectors of the $\left[g(\boldsymbol{\phi} \cdot \phi)^{2}-\sigma \phi_{1}^{2}\right]$ - and the $P(\phi)_{2} \otimes P(\phi)_{2}$-theories, i.e. the soliton-sectors of these theories are Poincarécovariant. This and Theorem 4 prove that the spectra of $P_{\pi}^{ \pm}$and $H_{\pi}^{ \pm}$are purely continuous (on each soliton-sector). This result seems to confirm that our construction of soliton-sectors is the correct one. The proof will appear elsewhere.

7) Final Remark. Due to several reasons the appearance of this paper has been delayed. The manuscript was, however, essentially complete before many of the recent papers about the quantum soliton appeared. Therefore we may not have given credit to this or the other author at places, where he might wish it. We apologize for that, but we have otherwise no reason to revise any parts of this paper. 
Acknowledgments. I thank A. S. Wightman for his stimulating interest in this work and useful conversations, and A. Casher for an interesting discussion.

\section{References}

1. Albeverio, S., Hoegh-Krohn, R.: Commun. math. Phys. 30, 171 (1972)

2. Araki, H.: Progr. Theor. Phys. 32, 844 (1964)

3. Coleman, S.: Quantum Sine-Gordon equation as the massive Thirring model, to appear in Phys. Rev. D.

4. Christ, N.H., Lee, T.D.: Quantum expansion of soliton solutions. Columbia University, Preprint 1975 (CO-2271-55)

5. Buchholz, D.: Collision theory for waves in two dimensions. CERN preprint TH. 2028-CERN

6. Bisognano, J., Wichman, E.: J. Math. Phys. 16, 985 (1975)

7. Borchers, H.-J.: Commun. math. Phys. 2, 49 (1966)

8. Dashen, R., Hasslacher, B., Neveu, A.: The particle spectrum in model field theories from semiclassical functional integral techniques. Inst. Adv. Study, Preprint (1975)

9. Doplicher, S., Haag, R., Roberts, J.: Commun. math. Phys. 23, 199 (1971); 35, 49 (1974)

10. Dobrushin, R., Minlos, R.: Funct. Anal. Appl. 7, 324 (1973); (English Translation)

11. Dunlop,F., Newman, C.: Multicomponent field theories and classical rotators, Indiana University, Preprint (1975)

12. Erice Summer School. In: Constructive quantum field theory, G. Velo, A. Wightman (eds.). Lecture notes in Physics 25. Berlin-Heidelberg-New York: Springer 1973

13. Ezawa, H., Swieca, J.: Commun. math. Phys. 5, 330 (1967)

14. Faddeev, L.: Quantization of solitons, Inst. Adv. Study, Preprint (1975); Faddeev, L., Takhtajan, A.: Theor. Math. Phys. 21, 160 (1974)

15. Fröhlich, J.: Phys. Rev. Letters 34, 833 (1975), and paper in preparation. See also the authors Erice lectures, 1975

16. Fröhlich, J.: Classical and quantum statistical mechanics in one and two dimensions: Two component Yukawa- and Coulomb systems. Commun. math. Phys. 47, 233-268 (1976)

17. Fröhlich, J., Seiler, E.: The massive Thirring-Schwinger model as the massive Sine-Gordon equation: Convergence of perturbation theory, Preprint (1976)

18. Fröhlich, J.: Schwinger functions and their generating functionals, II., to appear in Adv. Math.

19. Fröhlich, J.: The pure phases, the irreducible quantum fields. Princeton University, Preprint (1975)

20. Fröhlich, J.: Existence and analyticity in the bare parameters of the $\left[\lambda(\boldsymbol{\phi} \cdot \boldsymbol{\phi})^{2}-\sigma \phi_{1}^{2}-\mu \phi_{1}\right]$ quantum field models, in preparation

21. Goldstone, J., Jackiw, R.: Phys. Rev. D 11, 1486 (1975)

22. Glimm, J., Jaffe, A. : Ann. Math. 91, 362 (1970)

23. Glimm, J., Jaffe, A. : Acta Math. 125, 203 (1970)

24. Glimm, J., Jaffe, A.: Commun. math. Phys. 22, 1 (1971)

25. Guerra, F., Rosen,L., Simon, B.: Commun. math. Phys. 27, 10 (1972); 29, 233 (1973)

26. Glimm, J., Jaffe, A.: J. Math. Phys. 13, 1568 (1972)

27. Guerra, F., Rosen, L., Simon, B.: The $P(\phi)^{2}$ Euclidean quantum field theory as classical statistical mechanics. Ann. Math. 101, 111 (1975)

28. Glimm, J., Jaffe, A., Spencer, T.: Phase transitions for $\varphi_{2}^{4}$ quantum fields. Commun. math. Phys. 45, 203-216 (1975)

29. Hepp, K.: Commun. math. Phys. 1, 95 (1965)

30. Jost, R.: The general theory of quantized fields, Providence, R. I.: A.M.S. (1965); Streater, R., Wightman, A.: PCT, spin and statistics and all that. New York: Benjamin 1964

31. Lamb, G., Jr.: Rev. Mod. Phys. 43, 99 (1971)

32. Lanford, O., III.: In: Statistical mechanics and quantum field theory. Les Houches 1970, C. De Witt R. Stora (eds.), p. 1. New York: Gordon \& Breach 1971

33. May, R.: Phys. Lett. A 25, 282 (1967)

34. Osterwalder, K.: Commun. math. Phys. 29, 1 (1973); see also Refs. 1, 2, and 5 of this paper ${ }^{4}$

$4 \quad$ The original proof is due to H. Araki, Refs. 1,2 of 34. 
35. Polyakov, A.: Isomeric states of quantum fields, L. D. Landau, Inst. for Theor. Physics, Preprint (1974)

36. Simon, B.: The $P(\phi)_{2}$ Euclidean (quantum) field theory. Princeton series in Physics, Princeton University Press, Princeton (1974)

37. Simon, B., Griffiths, R.: Commun. math. Phys. 33, 145 (1973)

38. Spencer, T.: Commun. math. Phys. 39, 63 (1974)

39. Suzuki, M., Fisher, M.: J. Math. Phys. 12, 235 (1971)

40. Spencer, T.: The decay of the Bethe-Salpeter kernel in $P(\phi)_{2}$ quantum field models. Harvard University, Preprint (1975)

41. Sakai, S.: $C^{*}$-Algebras and $W^{*}$-Algebras, Ergebnisse der Mathematik und ihrer Grenzgebiete 60. Berlin-Heidelberg-New York: Springer 1971

42. Streater, R.F., Wilde, I. F.: Nucl. Phys. B 24, 561 (1970); see also Streater, R., Acta Phys. Austriaca, Suppl. XI, 317 (1973); Bonnard, P., Streater, R.: ZiF-University of Bielefeld, Preprint 1975

43. Takesaki, M.: Pacific J. Math. 34, 807 (1970)

Communicated by A. S. Wightman

Received October 1, 1975 